\title{
Increased Expression of UBE2T Predicting Poor Survival of Ovarian Cancer: Based on Comprehensive Analysis of UBE2s, Clinical Samples And the GEO Database
}

\section{Ruoyao Zou}

Shengjing Hospital of China Medical University

Haoya Xu

Shengjing Hospital of China Medical University

Feifei Li

Shandong Provincial Hospital

\section{Shengke Wang}

Shengjing Hospital of China Medical University

Liancheng Zhu ( $\nabla$ medecin@126.com )

Shengjing Hospital of China Medical University https://orcid.org/0000-0002-0814-5816

\section{Research article}

Keywords: UBE2T, UBE2s, ovarian cancer, prognostic value, bioinformatics analysis, immunohistochemistry

Posted Date: July 16th, 2020

DOI: https://doi.org/10.21203/rs.3.rs-41533/v1

License: (c) (i) This work is licensed under a Creative Commons Attribution 4.0 International License.

Read Full License

Version of Record: A version of this preprint was published at DNA and Cell Biology on January 1st, 2021. See the published version at https://doi.org/10.1089/dna.2020.5823. 


\section{Abstract}

Background囚Ubiquitin-conjugating enzymes E2 (UBE2) have been reported in the microenvironment of various malignant tumors, but their correlation with ovarian cancer remains elusive.

Methods: The Oncomine, GEPIA, Kaplan-Meier Plotter, cBioPortal, and STRING databases were used to systematically analyze the expression pattern, prognostic value, genetic variation, and biological function of 12 members of the UBE2 gene family in ovarian cancer. UBE2T exhibited the greatest correlation with ovarian cancer and was thus further examined. Gene set enrichment analysis (GSEA), as well as analyses of function and pathway enrichment, somatic mutations, copy number variation, and methylation were performed, and the correlation with immune cell infiltration was examined to explore the mechanism underlying aberrant UBE2T expression. Finally, the expression and prognostic value of UBE2T in ovarian cancer were verified by immunohistochemical evaluation of 131 clinical ovarian samples and the Gene Expression Omnibus (GEO) database (GSE51088, GSE73614, and GSE63885 datasets) analysis.

Results: The mRNA levels of UBE2C, UBE2N, UBE2S, and UBE2T were significantly upregulated in ovarian cancer compared with those in normal ovarian tissue. In patients with ovarian serous carcinoma, UBE2A, $U B E 2 B, U B E 2 C, U B E 2 G$, and $U B E 2 T$ upregulation and $U B E 2 R 2$ downregulation were associated with poor overall survival. Moreover, UBE2A, UBE2N, and UBE2T upregulation and UBE2G and UBE2R2 downregulation were associated with poor progression-free survival. Immunohistochemistry revealed that UBE2 $T$ was significantly upregulated in ovarian malignant tumors compared with that in borderline tumors, benign tumors, and normal ovarian tissues, and its high expression was associated with poor prognosis. The Cox model showed that UBE2T upregulation was an independent risk factor affecting the prognosis of ovarian cancer (hazard ratio: 4.095, $P=0.029$ ). The above results were verified in the GEO database. In addition, UBE2T was associated with specific immune cells and mainly involved in cell cycle-related events. Genomic analysis showed that TP53 and TTN mutations were associated with UBE2T expression. Gene copy number amplification and hypomethylation may be responsible for UBE2T upregulation in ovarian cancer.

Conclusions: UBE2 family members may play a role in the development of ovarian cancer. Specifically, UBE2T could serve as a new prognostic marker and therapeutic target for this disease.

\section{Background}

Ovarian cancer (OC) is the most deadly malignancy of the female reproductive system and the fifth leading cause of cancer-related death among women worldwide[1]. More than $70 \%$ of OC patients are diagnosed with advanced disease due to lack of typical clinical symptoms and effective diagnostic methods, which explains the high mortality rate of this disease. Despite major advances in surgical techniques, chemotherapy, and immunotherapy, the current treatments are still unsatisfactory, as the fiveyear overall survival (OS) rate is approximately $30 \%[2]$. Hence, the identification of biomarkers with high sensitivity and specificity, as well as the comprehension of their role in $\mathrm{OC}$, are urgently needed. 
The tumor microenvironment consists of multiple cellular and non-cellular components that support tumor growth and suppress antitumor response. The role of this system in tumor progression and its relevance for cancer immunotherapy have been extensively demonstrated[3]. Our previous analysis of genome-wide gene expression changes in HE4-transfected OC cells showed that ubiquitination is strongly involved in the development of malignant behavior. Recent findings have also revealed that ubiquitination-related enzymes are important components of the tumor microenvironment. These enzymes include ubiquitin-activating (UBE1), ubiquitin-conjugating (UBE2), and ubiquitin-ligating (UBE3) enzymes, which facilitate ubiquitination and lead to proteasome-mediated protein degradation[4]. The UBE2 family is composed of 40 members, which are considered as pivotal factors in the ubiquitination cascade[5]. Some studies have reported that aberrant expression of UBE2s in tumors has an impact on tumor prognosis, suggesting that UBE2s are informative tumor markers for early diagnosis and prediction of prognosis[6-8]. However, the biological role and action mechanism of these enzymes in $\mathrm{OC}$ have not been fully elucidated. UBE2T, a member of the UBE2 family, was the first to be defined as a key factor in the Fanconi anemia pathway[9]. Several studies have confirmed that UBE2T plays a carcinogenic role in various types of cancer, including hepatocellular carcinoma, as well as lung, breast, stomach, bladder, and prostate cancer, but its expression level and prognostic value in $O C$ are still unclear[10-15].

In this study, we selected 12 gene members of the UBE2 family with a possible relationship with OC, and used bioinformatics analysis to evaluate their expression and prognostic value. UBE2T exhibited the greatest correlation with $\mathrm{OC}$, which was verified by immunohistochemistry (IHC) and GEO database analysis. Moreover, the molecular function of UBE2T was explored. Our study provided valuable hints for the design of a new targeted therapy for OC.

\section{Methods}

\section{Oncomine database analysis}

Oncomine (http://www. oncomine. org) is a web-based gene chip data-mining platform consisting of microarray databases covering 19 types of human cancer. It includes 715 tumor microarrays, as well as 86,733 cancer and normal tissue samples[16]. Oncomine can be used to identify genes with differential expression in cancers and their respective normal tissues. We used Oncomine to analyze the mRNA expression of UBE2s in different types of cancer. The standardized normalization and parameters were as follows: $P$ value $<0.01$, fold change $>2$, and gene ranking in the top $10 \%$.

\section{GEPIA dataset analysis}

Gene Expression Profiling Interactive Analysis (GEPIA) (http://gepia.cancer-pku. cn/) is a database of RNA sequencing expression data from The Cancer Genome Atlas (TCGA) and Genotype-tissue Expression dataset (GTEx) projects, including 33 tumor types, 9,736 tumor samples, and 8,587 normal samples[17]. In this study, we used GEPIA to verify the differential expression of UBE2s in OC and normal ovarian tissues. In addition, the database was used to evaluate the correlations between different UBE2 members. $P<0.05$ indicated statistically significant differences. 


\section{GEO dataset acquirement}

Three distinct OC-related datasets (GSE51088, GSE73614, and GSE63885) and the corresponding clinical information were retrieved from the GEO database (https://www.ncbi.nlm.nih.gov/geo/). GSE51088, based on the GPL7264 platform (Agilent-012097 Human 1A Microarray (V2) G4110B), contained 140 epithelial ovarian malignant tumor samples, 12 borderline ovarian tumor samples, 5 benign ovarian tumor samples, and 15 normal ovarian samples[18]. GSE73614, obtained with the GPL6480 platform (Agilent-014850 Whole Human Genome Microarray 4× 44K G4112F), contained 107 epithelial ovarian cancer (EOC) samples[19]. GSE63885, based on the GPL570 platform (HG-U133_Plus_2; Affymetrix Human Genome U133 Plus 2.0 Array), contained 70 serous and 5 nonserous OC samples with complete clinical data[20]. All gene expression data were subjected to log2 transformation.

\section{TCGA data extraction and analysis}

Somatic mutations data corresponding to UBE2T high-low expression samples were downloaded from the TCGA-OV database. Data regarding OC-related gene copy number variations were downloaded from the cBioPortal (http://www.cbioportal.org/), and the samples were divided into four groups according to the copy number as follows: single deletions, diploid normal copy, low-amplification and highamplification. Wilcoxon test was used to compare the expression of UBE2T between two groups. UBE2 genetic variations in ovarian serous cystadenocarcinoma (such as amplifications, deep deletions, fusions, and mutations) were also analyzed in the CBioPortal database. OC methylation data were obtained from the Xena browser (https://xenabrowser.net/datapages/). Pearson correlation analysis was applied to evaluate the correlation between the methylation level and the expression of UBE2T.

\section{Kaplan-Meier plotter analysis}

The Kaplan-Meier plotter (http://kmplot. com/analysis) contains information on 54,675 genes and 10,188 cancer samples, including breast $(n=6234)$, lung $(n=3452)$, ovarian $(n=2190)$, and gastric $(n=1440)$ cancer. This tool is used to verify the impact of biomarker genes identified from GEO, TCGA, and the Cancer Biomedical Informatics Grid project on survival [21]. In this study, the Kaplan-Meier plotter was used to evaluate the prognostic value of different UBE2 members. OC patients were separated into a highexpression or a low-expression group, the best cutoff values were determined by algorithms embedded in KM plotter. The relevant hazard ratios (HRs), 95\% confidence intervals (Cls), and log-rank $P$ values were calculated.

\section{String protein network analysis}

The Search Tool for the Retrieval of Interacting Genes/Proteins (STRING) database (https://string-db. org/) is an effective tool for the analysis of functional associations between proteins. It contains information on 9.64 million proteins and 13.8 million interactions in 2,031 species[22]. We used the STRING database to predict the upstream and downstream regulatory proteins of UBE2s and the regulatory relations between these proteins in Homo sapiens. Interactions with a combined score $>0.7$ (high confidence) were considered significant. This information was used to construct a protein-protein 
interaction (PPI) network, and the Gene Ontology (GO) biological process and Kyoto Encyclopedia of Genes and Genomes (KEGG) pathway enrichment were analyzed by ClueGO and CluePedia plug-ins in Cytoscape3.2.1.

\section{Metascape enrichment and GSEA analysis}

The Metascape resource (http://metascape.org/gp/index. html) was utilized to predict the potential biological functions of target genes[23]. We used the cBioPortal database to identify genes that were coexpressed with UBE2T. The co-expressed genes with Spearman's correlation coefficient $\geq 0.35$ or $<-0.35$ were imported into Metascape for GO and KEGG pathway enrichment analysis. In the TCGA-OV database, 379 OC cases were divided into a high- and a low-expression group according to the median expression value of UBE2T. GSEA 3.0 software was used to identify the potential hallmark between the two expression groups by using default weighted enrichment statistics. "h.all.v6.2.symbols.gmt" was set as gene set database, and gene permutations were set to 1000 .

\section{TISIDB immune analysis}

The tumor-immune system interactions (TISIDB) database (http://cis. hku. hk/TISIDB) explores the function of genes and their role in tumor-immune interactions, and contains data on 998 genes related to antitumor immunity in 30 TCGA cancer types[24]. We employed the TISIDB database to predict correlations between UBE2T expression and tumor immune infiltrating cells, immune modulators, and major histocompatibility complex (MHC) molecules in OC.

\section{Participants and specimens}

A total of 131 ovarian paraffin-embedded tissue samples, surgically removed from inpatients at the Shengjing Hospital of China Medical University from 2008 to 2014, were collected. Pathological diagnosis was confirmed by two pathologists from the Department of Pathology. All patients were divided into 4 groups, including 86 cases of ovarian epithelial malignant tumors (all cases were primary $\mathrm{OC}$ and none of the patients received chemotherapy or hormone treatment before surgery; clinical information on all patients was complete), 20 cases of ovarian epithelial borderline tumors, 15 ovarian epithelial benign tumors, and 10 normal ovarian tissues. The median ages of patients in the above 4 groups were 53 (19-78), 44 (26-87), 46 (28-67), and 43 (32-62) years, respectively. The age differences between groups were not statistically significant $(P>0.05)$. The study was approved by the Clinical Research Ethics Committee of Shengjing Hospital of China Medical University, and all patients signed an informed consent.

\section{Immunohistochemistry}

The rabbit anti-human polyclonal antibody against UBE2T(10105-2-AP, 1:75) was purchased from Proteintech (Chicago, IL, USA), and the Streptavidin-peroxidase (SP) immunohistochemistry kit was purchased from Zsbio (Beijing, China). Ovarian tissues were fixed in $10 \%$ formalin, and $5-\mu m$ thick paraffin sections were prepared. The SP method was used to detect the expression of UBE2T. The presence of brownish-yellow staining on the cell membrane and in the cytoplasm was indicative of 
UBE2T positivity. A lung cancer sample was used as a positive control and phosphate-buffered saline was used instead of the antibody as a negative control. The staining intensity was classified as negative, light-yellow, brownish-yellow, and dark-brown, and scored 0, 1, 2, and 3, respectively. Based on the percentage of positive cells, the following categories were created: $<5 \%, 5 \%-25 \%, 26 \%-50 \%, 51-75 \%$, and $>$ $75 \%$, which scored $0,1,2,3$, and 4 , respectively. The final score was the product of the cell staining intensity score and the positive cell rate score: $0-2$ was negative $(-), 3-4$ weakly positive $(+), 5-8$ moderately positive (++), and 9-12 strongly positive (+++). Negativity and weak positivity were considered indicative of low expression, whereas moderate and strong positivity corresponded to high expression. To minimize the errors, each tissue section was independently reviewed by two observers. Inconsistent results were reviewed by a third observer.

\section{Statistical analysis}

SPSS 22.0 software (SPSS Inc., Chicago, IL, USA) was used for statistical analysis, GraphPad Prism 8.0 (La Jolla, CA, USA) and "survminer" R package were employed to generate figures. Chi squared and Fisher's exact tests were used to analyze counting data, while Student's $t$-test was used to analyze measurement data. Kaplan-Meier survival analysis and log-rank tests of the GEO database (GSE63885 and GSE73614) were used to assess the influence of UBE2T on OC prognosis, and the GSE51088 dataset was used to compare the expression of UBE2T in different groups. Univariate and multivariate analyses were based on Cox proportional hazard regression models. $P$ values $<0.05$ were defined as indicative of statistical significance. The patients with malignant $\mathrm{OC}$ were followed up, and inpatient data and telephone contacts were available. The last date of follow-up was November 30, 2019, and OS was defined as the time interval between the date of surgery and the date of death or follow-up endpoint.

\section{Results}

\section{Expression levels of UBE2 family members in patients with ovarian cancer}

We selected 12 gene members of the UBE2 family based on their possible association with OC, and used the Oncomine database to analyze their mRNA expression levels in different cancer and normal tissue samples (Fig. 1a and Table 1). The database contained a total of 433, 467, 450, 299, 439, 452, 409, 444, $314,392,299$, and 443 analyses for UBE2A, UBE2B, UBE2C, UBE2F, UBE2G, UBE2I, UBE2M, UBE2N, $U B E 2 R 2, U B E 2 S, U B E 2 T$, and $U B E 2 V 2$, respectively. Oncomine analysis revealed that the $m R N A$ expression of UBE2C, UBE2M, UBE2N, UBE2S, UBE2T, and UBE2V2 was upregulated in patients with OC. UBE2C mRNA levels were significantly higher in $8 O C$ datasets: in Yoshihara's dataset[25], UBE2C was significantly upregulated in 43 cases of ovarian serous adenocarcinoma compared with that in 10 normal tissue samples $(P=5.73 \mathrm{E}-13$, fold change $=12.955)$; in Lu's dataset[26], UBE2C was overexpressed in ovarian serous adenocarcinoma, endometrioid adenocarcinoma, and clear cell adenocarcinoma $(P=$ $1.39 \mathrm{E}-7$, fold change $=2.358 ; P=1.43 \mathrm{E}-4$, fold change $=2.298 ; P=0.001$; fold change $=2.156$ ); in the TCGA, Adib's, and Welsh's dataset[27, 28], UBE2C was highly expressed in ovarian serous carcinoma compared with that in normal tissues. $(P=2.24 \mathrm{E}-7$, fold change $=10.184 ; P=0.004$, fold change $=3.828$; $P=8.50 \mathrm{E}-8$; fold change $=4.020$, respectively). Similar results were reported by Bonome et al . in ovarian 
carcinoma $(P=2.50 \mathrm{E}-10$, fold change $=4.182)[29]$. Moreover, in Bonome's dataset[29], UBE2M $(P=1.63 \mathrm{E}-$ 7 , fold change $=2.479), U B E 2 N(P=6.86 \mathrm{E}-10$, fold change $=2.111)$, and UBE2S $(P=5.22 \mathrm{E}-11$, fold change $=3.855)$ were upregulated in OC. In the TCGA dataset, UBE2S $(P=1.53 \mathrm{E}-5$, fold change $=3.267)$ and UBE2V2 $(P=7.04 \mathrm{E}-8$, fold change $=2.031)$ were upregulated in ovarian serous cystadenocarcinoma. Significant UBE2T overexpression compared with that in normal tissues was found in Yoshihara's dataset (ovarian serous adenocarcinoma; $P=4.30 \mathrm{E}-8$, fold change $=8.877$ )[25], and in Lu's dataset (ovarian serous adenocarcinoma and endometrioid adenocarcinoma; $P=3.64 \mathrm{E}-8$, fold change $=2.751$ and $P=$ $2.19 \mathrm{E}-6$, fold change $=2.814$, respectively) [26] In addition, the mRNA levels of UBE2B, UBE2G, and UBE2I were reduced in patients with OC. In Bonome's dataset[29], UBE2B $(P=2.98 \mathrm{E}-9$, fold change $=-2.405)$, UBE2G $(P=6.86 \mathrm{E}-10$, fold change $=-2.111)$, and UBE2I $(P=8.72 \mathrm{E}-8$, fold change $=-3.355)$ were downregulated in ovarian carcinoma compared with those in ovarian surface epithelium. A similar trend was found for UBE2/ in Welsh's [28] and TCGA datasets: the mRNA levels of UBE2/ in ovarian serous surface papillary carcinoma $(P=8.10 \mathrm{E}-5$, fold change $=-3.672)$ and ovarian serous cystadenocarcinoma $(P=2.93 \mathrm{E}-7$, fold change $=-2.884)$ were significantly lower than those in normal tissues. Moreover, Oncomine analysis showed that the mRNA expression of UBE2A, UBE2F, and UBE2R2 was not significantly different between $\mathrm{OC}$ and normal tissues.

Next, we used the GEPIA database to compare the mRNA levels of different UBE2s in OC and normal ovarian tissues (Fig. 1b). The results indicated that the expression levels of UBE2C, UBE2F, UBE2N, $U B E 2 S$, and $U B E 2 T$ were significantly higher in $O C$ tissues than in normal ovarian tissues, whereas those of UBE2A, UBE2B, UBE2G, UBE2I, UBE2M, UBE2R2, and UBE2V2 were not significantly different between the two groups. Combined analysis of the GEPIA and Oncomine databases revealed that the expression of UBE2C, UBE2N, UBE2S, and UBE2T was upregulated in OC compared with that in normal tissues.

Figure 1 Differential expression of UBE2s in cancer. a The mRNA expression levels of different UBE2 members in various types of cancers (Oncomine). The numbers in the cells represent the data sets meeting the threshold. Red and blue cells represent upregulation and downregulation, respectively. The color intensity reflects the level of significance. $\mathbf{b}$ The mRNA levels of UBE2 members in OC and normal ovarian tissues (GEPIA). The box plots indicate UBE2 expression in 426 cases of OC (red) and 88 cases of corresponding normal tissues (gray). ${ }^{\star} P<0.01$ 
Table 1

UBE2 expression in different types of ovarian cancer and normal ovarian tissues (Oncomine)

\begin{tabular}{|c|c|c|c|c|c|c|}
\hline Gene & Dataset & Tumor (cases) & Normal (cases) & $\begin{array}{l}\text { Fold } \\
\text { change }\end{array}$ & t-test & $\begin{array}{l}P \\
\text { value }\end{array}$ \\
\hline UBE2B & Bonome & Ovarian Carcinoma (185) & $\begin{array}{l}\text { Ovarian } \\
\text { Surface } \\
\text { Epithelium (10) }\end{array}$ & -2.405 & -14.212 & $\begin{array}{l}2.98 \mathrm{E}- \\
9\end{array}$ \\
\hline \multirow[t]{8}{*}{ UBE2C } & Yoshihara & $\begin{array}{l}\text { Ovarian Serous } \\
\text { Adenocarcinoma (43) }\end{array}$ & $\begin{array}{l}\text { Peritoneum } \\
\text { (10) }\end{array}$ & 12.955 & 14.201 & $\begin{array}{l}5.73 \mathrm{E}- \\
13\end{array}$ \\
\hline & Lu & $\begin{array}{l}\text { Ovarian Serous } \\
\text { Adenocarcinoma (20) }\end{array}$ & $\begin{array}{l}\text { Ovarian } \\
\text { Surface } \\
\text { Epithelium (5) }\end{array}$ & 2.358 & 8.107 & $\begin{array}{l}1.39 \mathrm{E}- \\
7\end{array}$ \\
\hline & Lu & $\begin{array}{l}\text { Ovarian Endometrioid } \\
\text { Adenocarcinoma (9) }\end{array}$ & $\begin{array}{l}\text { Ovarian } \\
\text { Surface } \\
\text { Epithelium (5) }\end{array}$ & 2.298 & 5.260 & $\begin{array}{l}1.43 \mathrm{E}- \\
4\end{array}$ \\
\hline & Lu & $\begin{array}{l}\text { Ovarian Clear Cell } \\
\text { Adenocarcinoma (7) }\end{array}$ & $\begin{array}{l}\text { Ovarian } \\
\text { Surface } \\
\text { Epithelium (5) }\end{array}$ & 2.156 & 4.314 & 0.001 \\
\hline & TCGA & $\begin{array}{l}\text { Ovarian Serous } \\
\text { Cystadenocarcinoma } \\
\text { (586) }\end{array}$ & Ovary (8) & 10.184 & 16.188 & $2.24 \mathrm{E}-$ \\
\hline & Adib & $\begin{array}{l}\text { Ovarian Serous } \\
\text { Adenocarcinoma (6) }\end{array}$ & Ovary (4) & 3.828 & 4.280 & 0.004 \\
\hline & Welsh & $\begin{array}{l}\text { Ovarian Serous Surface } \\
\text { Papillary Carcinoma (28) }\end{array}$ & Ovary (4) & 4.020 & 13.503 & $\begin{array}{l}8.50 \mathrm{E}- \\
8\end{array}$ \\
\hline & Bonome & Ovarian Carcinoma (185) & $\begin{array}{l}\text { Ovarian } \\
\text { Surface } \\
\text { Epithelium (10) }\end{array}$ & 4.182 & 15.168 & $\begin{array}{l}2.50 \mathrm{E}- \\
10\end{array}$ \\
\hline UBE2G & Bonome & Ovarian Carcinoma (185) & $\begin{array}{l}\text { Ovarian } \\
\text { Surface } \\
\text { Epithelium (10) }\end{array}$ & -2.410 & -14.317 & $\begin{array}{l}6.69 \mathrm{E}- \\
11\end{array}$ \\
\hline
\end{tabular}

\section{Genetic alterations of UBE2 family members in patients with OC}

Genetic alterations of UBE2 members were analyzed by using the cBioPortal database. A total of 1680 cases from three datasets (489 cases from TCGA, Nature 2011; 585 cases from TCGA, PanCancer Atlas; and 606 cases from TCGA, Provisional) of ovarian serous cystadenocarcinoma were analyzed (Fig. 2a,b). The alteration rates were $28.47 \%, 19.86 \%$, and $13.5 \%$, in the 3 OC datasets, respectively. Twelve UBE2 genes showed various levels of genetic alteration. In all three datasets, only gene amplification was observed for UBE2C and UBE2V2; UBE2C exhibited the highest rate of amplification (6.35\% in TCGA), while the rate of UBE2V2 amplification was $4.46 \%$ in TCGA. Most genetic variations in UBE2 family genes were amplifications and deep deletions, while mutations were found in UBE2A $(0.17 \%$ in TCGA), UBE2M (0.17\% in TCGA and TCGA pub), and UBE2T $(0.17 \%$ in TCGA PanCan), and multiple alterations were found in UBE2A and UBE2I (both $0.17 \%$ in TCGA PanCan). In addition to gene amplification and deep deletions, UBE2G variations included gene fusions $(1.37 \%$ in TCGA). The Kaplan-Meier curve indicated that there was no significant difference in $O S$ and disease-free survival (DFS) regardless of the presence of alterations in one of the query genes ( $P$ values, 0.804 and 0.393 , respectively, Fig. 2c,d) 


\begin{tabular}{|c|c|c|c|c|c|c|}
\hline Gene & Dataset & Tumor (cases) & Normal (cases) & $\begin{array}{l}\text { Fold } \\
\text { change }\end{array}$ & t-test & $\begin{array}{l}P \\
\text { value }\end{array}$ \\
\hline \multirow[t]{3}{*}{ UBE2I } & TCGA & $\begin{array}{l}\text { Ovarian Serous } \\
\text { Cystadenocarcinoma } \\
(586)\end{array}$ & Ovary (8) & -2.884 & -12.936 & $\frac{2.93 \mathrm{E}-}{7}$ \\
\hline & Welsh & $\begin{array}{l}\text { Ovarian Serous Surface } \\
\text { Papillary Carcinoma (28) }\end{array}$ & Ovary (4) & -3.672 & -4.312 & $\begin{array}{l}8.10 \mathrm{E}- \\
5\end{array}$ \\
\hline & Bonome & Ovarian Carcinoma (185) & $\begin{array}{l}\text { Ovarian } \\
\text { Surface } \\
\text { Epithelium (10) }\end{array}$ & -3.355 & -12.246 & $\begin{array}{l}8.72 \mathrm{E}- \\
8\end{array}$ \\
\hline UBE2M & Bonome & Ovarian Carcinoma (185) & $\begin{array}{l}\text { Ovarian } \\
\text { Surface } \\
\text { Epithelium (10) }\end{array}$ & 2.479 & 9.829 & $\begin{array}{l}1.63 \mathrm{E}- \\
7\end{array}$ \\
\hline UBE2N & Bonome & Ovarian Carcinoma (185) & $\begin{array}{l}\text { Ovarian } \\
\text { Surface } \\
\text { Epithelium (10) }\end{array}$ & 2.111 & 12.184 & $\begin{array}{l}6.86 \mathrm{E}- \\
10\end{array}$ \\
\hline \multirow[t]{2}{*}{ UBE2S } & Bonome & Ovarian Carcinoma (185) & $\begin{array}{l}\text { Ovarian } \\
\text { Surface } \\
\text { Epithelium (10) }\end{array}$ & 3.855 & 14.375 & $\begin{array}{l}5.22 \mathrm{E}- \\
11\end{array}$ \\
\hline & TCGA & $\begin{array}{l}\text { Ovarian Serous } \\
\text { Cystadenocarcinoma } \\
\text { (586) }\end{array}$ & Ovary (8) & 3.267 & 8.944 & $\begin{array}{l}1.53 \mathrm{E}- \\
5\end{array}$ \\
\hline \multirow[t]{3}{*}{ UBE2T } & Lu & $\begin{array}{l}\text { Ovarian Endometrioid } \\
\text { Adenocarcinoma (9) }\end{array}$ & $\begin{array}{l}\text { Ovarian } \\
\text { Surface } \\
\text { Epithelium (5) }\end{array}$ & 2.814 & 8.153 & $\begin{array}{l}2.19 \mathrm{E}- \\
6\end{array}$ \\
\hline & Lu & $\begin{array}{l}\text { Ovarian Serous } \\
\text { Adenocarcinoma (20) }\end{array}$ & $\begin{array}{l}\text { Ovarian } \\
\text { Surface } \\
\text { Epithelium (5) }\end{array}$ & 2.751 & 7.789 & $\begin{array}{l}3.64 \mathrm{E}- \\
8\end{array}$ \\
\hline & Yoshihara & $\begin{array}{l}\text { Ovarian Serous } \\
\text { Adenocarcinoma (43) }\end{array}$ & $\begin{array}{l}\text { Peritoneum } \\
(10)\end{array}$ & 8.877 & 10.048 & $\begin{array}{l}4.30 \mathrm{E}- \\
8\end{array}$ \\
\hline
\end{tabular}

\section{Genetic alterations of UBE2 family members in patients with OC}

Genetic alterations of UBE2 members were analyzed by using the cBioPortal database. A total of 1680 cases from three datasets (489 cases from TCGA, Nature 2011; 585 cases from TCGA, PanCancer Atlas; and 606 cases from TCGA, Provisional) of ovarian serous cystadenocarcinoma were analyzed (Fig. 2a,b). The alteration rates were $28.47 \%, 19.86 \%$, and $13.5 \%$, in the 3 OC datasets, respectively. Twelve UBE2 genes showed various levels of genetic alteration. In all three datasets, only gene amplification was observed for UBE2C and UBE2V2; UBE2C exhibited the highest rate of amplification (6.35\% in TCGA), while the rate of UBE2V2 amplification was $4.46 \%$ in TCGA. Most genetic variations in UBE2 family genes were amplifications and deep deletions, while mutations were found in UBE2A $(0.17 \%$ in TCGA), UBE2M (0.17\% in TCGA and TCGA pub), and UBE2T $(0.17 \%$ in TCGA PanCan), and multiple alterations were found in UBE2A and UBE2I (both $0.17 \%$ in TCGA PanCan). In addition to gene amplification and deep deletions, UBE2G variations included gene fusions $(1.37 \%$ in TCGA). The Kaplan-Meier curve indicated that there was no significant difference in OS and disease-free survival (DFS) regardless of the presence of alterations in one of the query genes ( $P$ values, 0.804 and 0.393 , respectively, Fig. 2c,d) 


\begin{tabular}{|c|c|c|c|c|c|c|}
\hline Gene & Dataset & Tumor (cases) & Normal (cases) & $\begin{array}{l}\text { Fold } \\
\text { change }\end{array}$ & t-test & $\begin{array}{l}P \\
\text { value }\end{array}$ \\
\hline UBE2V2 & TCGA & $\begin{array}{l}\text { Ovarian Serous } \\
\text { Cystadenocarcinoma } \\
(586)\end{array}$ & Ovary (8) & 2.031 & 14.981 & $\begin{array}{l}7.04 \mathrm{E}- \\
8\end{array}$ \\
\hline \multicolumn{7}{|c|}{ Genetic alterations of UBE2 family members in patients with OC } \\
\hline \multicolumn{7}{|c|}{$\begin{array}{l}\text { Genetic alterations of UBE2 members were analyzed by using the cBioPortal database. A total of } \\
1680 \text { cases from three datasets ( } 489 \text { cases from TCGA, Nature } 2011 ; 585 \text { cases from TCGA, } \\
\text { PanCancer Atlas; and } 606 \text { cases from TCGA, Provisional) of ovarian serous cystadenocarcinoma } \\
\text { were analyzed (Fig. } 2 a, b) \text {. The alteration rates were } 28.47 \%, 19.86 \% \text {, and } 13.5 \% \text {, in the } 3 \text { OC datasets, } \\
\text { respectively. Twelve UBE2 genes showed various levels of genetic alteration. In all three datasets, only } \\
\text { gene amplification was observed for UBE2C and UBE2V2; UBE2C exhibited the highest rate of } \\
\text { amplification ( } 6.35 \% \text { in TCGA), while the rate of UBE2V2 amplification was } 4.46 \% \text { in TCGA. Most } \\
\text { genetic variations in UBE2 family genes were amplifications and deep deletions, while mutations were } \\
\text { found in UBE2A (0.17\% in TCGA), UBE2M (0.17\% in TCGA and TCGA pub), and UBE2T (0.17\% in } \\
\text { TCGA PanCan), and multiple alterations were found in UBE2A and UBE2I (both } 0.17 \% \text { in TCGA } \\
\text { PanCan). In addition to gene amplification and deep deletions, UBE2G variations included gene } \\
\text { fusions (1.37\% in TCGA). The Kaplan-Meier curve indicated that there was no significant difference in } \\
\text { OS and disease-free survival (DFS) regardless of the presence of alterations in one of the query genes } \\
\text { (P values, } 0.804 \text { and } 0.393 \text {, respectively, Fig. } 2 \text { c, d) }\end{array}$} \\
\hline
\end{tabular}

Figure 2 Frequency of UBE2 gene alterations in OC (cBioPortal). a Left, OncoPrint visual summary of alterations in UBE2 family members; right, summary of genetic alteration frequency in UBE2 genes. $\mathbf{b}$ Analysis of genetic alteration frequency in UBE2 family members in three datasets (TCGA, TCGA PanCan, and TCGA pub). The alterations included amplification (red), mutations (green), fusions (purple), deep deletions (blue), and multiple alterations (grey). c Kaplan-Meier plots comparing OS in cases with and without UBE2 gene alterations. $\mathbf{d}$ Kaplan-Meier plots comparing DFS in cases with and without UBE2 gene alterations

\section{Prognostic value of UBE2 genes in patients with ovarian cancer}

Kaplan-Meier plotter analysis was applied to assess the relationship between the mRNA expression of individual UBE2 members and progression-free survival (PFS) in 1436 clinical OC patients (Fig. 3). We found that the mRNA levels of UBE2A, UBE2B, UBE2C, UBE2G, UBE2N, UBE2R2, and UBE2T were associated with $O C$ prognosis, while those of the remaining members were not. Increased mRNA levels of $U B E 2 A, U B E 2 B, U B E 2 C, U B E 2 N$, and $U B E 2 T$, and decreased $m R N A$ levels of UBE2G and UBE2R2 were significantly associated with poor prognosis.

Ovarian serous tumors represent the most common histological subtype among ovarian tumors. The prognostic value of UBE2 genes with expression levels significantly correlated with OC prognosis was further investigated in ovarian serous tumors. Increased mRNA levels of UBE2A (Fig. 4a), UBE2B (Fig. 4b), $U B E 2 C$ (Fig. 4c), UBE2G (Fig. 4d), and UBE2T (Fig. 4g) were significantly correlated with poor OS in patients with ovarian serous tumors. Interestingly, increased expression of UBE2A (Fig. 5a) and UBE2G (Fig. 5d) was associated with shorter OS but longer PFS. Moreover, UBE2N (Fig. 5e) and UBE2T (Fig. 5g) upregulation was significantly correlated with poor PFS in patients with ovarian serous tumors, while 
UBE2R2 downregulation (Fig. 4f, 5f) was correlated with poor OS and PFS. Based on the above results, in patients with ovarian serous tumors, the expression levels of UBE2R2 and UBE2T were predictors of favorable and poor prognosis, respectively.

By integrating the results of Oncomine, GEPIA, and KM plotter, we observed that UBE2T was significantly upregulated in ovarian cancer compared to normal tissues, and that high UBE2T mRNA expression was significantly correlated with shorter OS and PFS in patients with ovarian serous tumors. Moreover, we assessed the prognostic value of UBE2T in relation to the pathological grade, FIGO stage, and TP53 status of ovarian serous tumors. Increased expression of UBE2T was correlated with poor OS in patients with tumors of all FIGO stages $(P<0.05$, Fig. $6 \mathrm{c}, \mathrm{d})$. Moreover, UBE2T upregulation predicted poor OS in patients with both mutated and wild-type TP53 $(P<0.05$, Fig. 6e,f). Although among patients with well/moderate and poor differentiation, those with high UBE2T expression tended to have shorter OS compared to patients with low UBE2T expression, the difference was not statistically significant $(P>0.05$, Fig. 6a,b).

Figure 3 Prognostic value of UBE2 mRNA levels in patients with OC (PFS in Kaplan-Meier plotter).. a-I Prognostic significance of individual UBE2 members in OC. Red line means high expression and black line means low expression. The $P$ values were calculated using the log-rank test. ${ }^{\star} P<0.05$

Figure 4 Prognostic value of UBE2 mRNA levels in patients with ovarian serous tumors (OS in KaplanMeier plotter). a-g Prognostic significance of individual UBE2s in ovarian serous tumors. $\mathbf{h}$ Prognostic HRs of individual UBE2s in ovarian serous tumors. Red line means high expression and black line means low expression. The $P$ values were calculated using the log-rank test. ${ }^{\star} P<0.05$

Figure 5 Prognostic value of UBE2 mRNA levels in patients with ovarian serous tumors (PFS in KaplanMeier plotter). a-g Prognostic significance of individual UBE2s in ovarian serous tumors. $\mathbf{h}$ Prognostic HRs of individual UBE2s in ovarian serous tumors. Red line means high expression and black line means low expression. The $P$ values were calculated using the log-rank test. ${ }^{*} P<0.05$

\section{Analysis of the interactions between UBE2 family members}

Spearman's correlation coefficients were calculated by using the GEPIA platform to investigate the relationships between the expression levels of different UBE2 members in OC (Fig. 6g). The coefficients ranged from 0.013 (UBE2B vs. UBE2M) to 0.67 (UBE2C vs. UBE2S). The results indicated a moderate positive correlation between the expression levels of UBE2A, UBE2N, UBE2S, UBE2T, and UBE2V2 $(0.3<\mathrm{r}$ $\leq 0.7$ ), and a low positive correlation between the expression levels of $U B E 2 C, U B E 2 F, U B E 2 M$, and UBE2R2 $(0<\mathrm{r} \leq 0.3)$. However, UBE2B showed a weakly positive correlation with other UBE2 genes, in addition to a weakly negative correlation with UBE2C ( $\mathrm{r}=-0.017)$.

A protein-protein interaction (PPI) network consisting of 12 UBE2 family members and 20 UBE2interacting proteins was visualized by using Cytoscape (PPI enrichment $P$ value $<1.0 \mathrm{E}-16$ ). Among 32 nodes, the most significant 10 proteins were UBA2, UBC, NEDD8, RAD18, SUMO1/2, RANBP2, RABGAP1, 
$A N A P C 11$, and $C D C 20$. Biological process analysis showed that UBE2 proteins were mainly involved in anaphase-promoting complex-dependent catabolic processes, protein neddylation, protein sumoylation, postreplication repair, and positive regulation of ubiquitin protein ligase activity (Fig. 6h). Pathway analysis showed that the UBE2 enzymes were mainly implicated in ubiquitin-mediated proteolysis, cell cycle, oocyte meiosis, and progesterone-mediated oocyte maturation (Fig. 6i, Additional file 1).

Figure 6 Prognostic significance of UBE2T expression level in ovarian serous tumors of different pathological grade (a,b), FIGO stage (c,d), and TP53 mutational status (e,f) (OS in Kaplan-Meier plotter). Analysis of the interactions between UBE2 family members (GEPIA and Cytoscape).

g Spearman's correlation coefficients between UBE2 family members. Red and blue cells represent positive and negative correlations, respectively. The color intensity reflects the strength of the correlations. $\mathbf{h}$ GO biological process enrichment analysis of UBE2s and their interactors. i KEGG pathway analysis of UBE2s and their interactors. ${ }^{*} P<0.05$

\section{Molecular mechanisms related to UBE2T expression in ovarian cancer}

A total of 536 genes were obtained from the cBioPortal database, based on Spearman's correlation coefficients higher than 0.35 between their expression and that of UBE2T. Information about the coexpressed genes is shown in Additional file 2. The Metascape portal was used, and $P<0.01$ was set as the threshold value to screen the top GO annotations and KEGG pathway results regarding UBE2T and its interactors. The top $20 \mathrm{GO}$ enrichment items were classified into three functional groups: biological process (BP, 13 items), molecular function (MF, 1 items), and cellular component (CC, 6 items) (Fig. 7a). Regarding the $\mathrm{BP}$, the genes co-expressed with UBE2T were mainly involved in cell division, cell cycle phase transition, DNA replication, DNA repair, and DNA conformation changes. Based on the MF, the identified genes were mainly associated with catalytic activity, acting on DNA. In terms of CC, the genes were enriched in the following categories: chromosomal region, nuclear chromosome, spindle, microtubule organizing center, and replication fork. The top 14 KEGG pathways for genes that were coexpressed with UBE2T are shown in Fig. 7b. Among these, cell cycle signaling, DNA replication, spliceosome, Fanconi anemia, and p53 signaling pathways were found to be involved in OC tumorigenesis and progression. To further determine the relationship between the enriched terms, a similarity network was constructed, where terms with a similarity $>0.3$ were connected by edges. The network was visualized by using Cytoscape. Each node represented an enriched term and was colored according to its cluster (Fig. 7C), and $P$-value (Fig. 7d). In patients with high UBE2T expression, significantly upregulated gene sets with nominal $P<0.05$ and FDR $<0.25$ included "HALLMARK_E2F_TARGETS" and "HALLMARK_G2M_CHECKPOINT". The enrichment plots are shown in Fig. 7e.

The presence of somatic mutations was investigated in cases with high and low UBE2T expression. TP53 and TTN were the top two mutated genes in both groups, and a high frequency of mutations in DST, CSMD1, MUC17, and NEB genes was specifically found in patients with high UBE2T expression. Moreover, mutations in TOP2A, VPS13B, NF1, AHNAK, and FLG2 were significantly enriched in patients with low 
UBE2T expression (Fig. 7f,g). KEGG pathway analysis further demonstrated that in patients with high UBE2T expression the mutations mainly affected focal adhesion, calcium signaling, and ECM-receptor interactions (Fig. 7h). To explore the cause of UBE2T upregulation in OC, we analyzed its correlation with gene copy number and methylation level. We found that UBE2T expression increased with the copy number. Therefore, the high level of UBE2T could be partially attributed to gene amplification (Fig. 7i). The expression of UBE2T was negatively correlated with the methylation level, as hypomethylation was associated with high UBE2T expression (Pearson's (372) $=-0.125, P=0.0162$, Fig. 7j).

Figure 7 Molecular mechanisms related to UBE2T expression in ovarian cancer (Metascape). a Bar graph of GO enriched terms colored by $P$-values. b Bar graph of KEGG enriched terms colored by $P$-values. $\mathbf{C}$ Network of enriched terms colored according to clusters: terms belonging to the same cluster are more closely related to each other. $\mathbf{d}$ Network of enriched terms colored according to $P$-value: the higher the number of genes in each term, the higher the statistical significance. e Gene sets significantly enriched in patients with high UBE2T expression (HALLMARK_E2F_TARGETS and HALLMARK_G2M_CHECKPOINT). f Top 20 somatically mutated genes in patients with high UBE2T expression. $\mathbf{g}$ Top 20 somatically mutated genes in patients with low UBE2T expression. $\mathrm{h} \mathrm{KEGG}$ pathway analysis in the UBE2T high expression mutation group. $\mathbf{i}$ Analysis of the correlation between copy number variation and UBE2T expression. $\mathbf{j}$ Analysis of the correlation between methylation and UBE2T expression

\section{Correlation between immune factors and UBE2T expression in ovarian cancer}

Recently, immunotherapy has received increasing attention, becoming a new treatment strategy for ovarian cancer. We further explored the correlation between immune factors, including tumor-infiltrating lymphocytes (TILs) and immune modulators, and UBE2T expression in OC by using the TISIDB database. Immune modulators can be further classified into immune inhibitors, immune stimulators, and $\mathrm{MHC}$ molecules. TILs with the highest correlation with UBE2T expression included act_CD 4 cells (Spearman: rho $=0.331, P=3.57 \mathrm{e}-09$ ), eosinophils (Spearman: rho=-0.424, $P<2.2 \mathrm{E}-16)$, neutrophils (Spearman: rho $=-0.274, P=1.23 \mathrm{E}-06$ ), and memory B cells (Spearman: rho $=-0.271, P=1.61 \mathrm{E}-06)$ (Fig. 8b). Immune inhibitors with the highest correlation with UBE2T expression included CSF1R (Spearman: rho $=-0.345, P=$ 6.53E-10), TGFB1 (Spearman: rho $=-0.257, P=5.53 \mathrm{E}-06), K D R$ (Spearman: rho $=-0.228, P=5.95 \mathrm{E}-05$ ), and CD160 (Spearman: rho $=-0.155, P=0.0064$ ) (Fig. 8d). Immune stimulators with the strongest correlation with UBE2T expression included C10orf54 (Spearman: rho=-0.336, $P=1.87 \mathrm{E}-09)$, NTSE (Spearman: rho $=-0.308, P=4.13 \mathrm{E}-08$ ), TNFSF14 (Spearman: rho $=-0.298, P=1.23 \mathrm{E}-07$ ), and TNFSF15 (Spearman: rho $=-0.257, P=5.37 \mathrm{E}-06$ ) (Fig. 8f). MHC molecules most strongly correlated with UBE2T expression included HLA-E (Spearman: rho=-0.173, $P=0.00245)$, HLA-DOA (Spearman: rho $=-0.171, P=0.00265)$, HLA-DQB1 (Spearman: rho $=-0.129, P=0.0236$ ), and HLA-DQA1 (Spearman: rho $=-0.121, P=0.0341$ ) (Fig. 8h). Therefore, UBE2T may affect the immune activity in the $\mathrm{OC}$ microenvironment by regulating the above immune factors.

Figure 8 The correlation between immune factors and UBE2T in ovarian cancer determined by TISIDB analysis. a Correlation between UBE2T expression and tumor-infiltrating lymphocytes (TILs). b TILs with 
the highest correlation with UBE2T expression. c Correlation between UBE2T expression and immune inhibitors. $\mathbf{d}$ Immune inhibitors showing the highest correlation with UBE2T expression. e Correlation between UBE2T expression and immune stimulators. $\mathrm{f}$ Immune stimulators showing the highest correlation with UBE2T expression. $\mathbf{g}$ Correlation between UBE2T expression and MHC molecules. $\mathbf{h}$ MHC molecules exhibiting the highest correlation with UBE2T expression. Red and blue cells indicate positive and negative correlations, respectively. The color intensity reflects the strength of the correlation.

\section{UBE2T expression in different ovarian tissues}

IHC staining demonstrated that UBE2T was mainly localized in the cytoplasm and the plasma membrane. The rates of positive and highly positive expression of UBE2T in ovarian epithelial malignant tumors (89.53\% and $72.09 \%$, respectively) were significantly higher than in ovarian epithelial borderline tumors (55.00\% and $25.00 \%$, respectively), ovarian epithelial benign tumors $(26.37 \%$ and $13.34 \%$, respectively), and normal ovarian tissues $(20.00 \%$ and $10.00 \%$, respectively, Fig. 9e,f). Consistently, GSE51088 analysis results showed a significant upregulation of UBE2T in malignant tumors compared to borderline tumors, benign tumors, and normal ovary (Fig. 9g).

Figure 9 UBE2T expression in different ovarian tissues. Immunohistochemical detection of UBE2T in OC tissues (a), borderline tumors (b), benign tumors (c), and normal ovarian tissues (d) (magnification, left $\times$ 200 , right $\times 400$ ). e Positive rates of UBE2T expression in clinical samples of different groups. $f$ Highly positive rates of UBE2T expression in clinical samples of different groups. $\mathbf{g} U B E 2 T$ expression in the different groups, according to the GSE51088 dataset. $\mathbf{h}$ Correlation between UBE2T expression and overall survival in 86 patients, based on clinical samples. i Correlation between UBE2T expression and overall survival in 107 samples, according to the GSE73614 dataset. $\mathbf{j}$ Correlation between UBE2T expression and overall survival in 75 samples, according to the GSE63885 dataset. ${ }^{\star} P<0.05, * \star P<0.01$, $\star \star \star P<0.001, * \star \star \star * P<0.0001$

\section{Relationship between UBE2T expression and clinicopathological features of epithelial ovarian cancer}

According to the median expression value of UBE2T, OC patients in GSE datasets were divided into a high-expression and a low-expression group. We found a significantly higher expression of UBE2T in FIGO stage III-IV patients than in FIGO stage I-II patients in both clinical samples $(85.11 \%$ vs. $56.41 \%, P$ $=0.003)$ and GSE73614 (61.02\% vs. $37.50 \%, P=0.016)$. However, although UBE2T expression did not significantly correlate with age, stage of differentiation, presence of lymph node metastasis or pathologic subtype in our clinical samples, it was strongly associated with the pathological subtype of OC in GSE73614 ( $P=0.001$, Table 2, 3). UBE2T displayed the highest high positive rate in serous carcinoma $(100 \%)$ and the lowest high positive rate in clear cell carcinoma $(27.03 \%)$. 
Table 2

Correlation between UBE2T expression and clinicopathological features in ovarian cancer clinical samples

\begin{tabular}{|c|c|c|c|c|c|c|c|}
\hline Characteristics & Cases & Low & & High & & $\begin{array}{l}\text { High } \\
\text { nositive rate }\end{array}$ & Chi square \\
\hline & $\begin{array}{l}(n= \\
86)\end{array}$ & - & + & ++ & +++ & $(\%)$ & (P value) \\
\hline Age (years) & & & & & & & $\begin{array}{l}0.031 \\
(0.861)\end{array}$ \\
\hline$₫ 60$ & 67 & 9 & 10 & 26 & 22 & 71.64 & \\
\hline$\geq 60$ & 19 & 0 & 5 & 7 & 7 & 73.68 & \\
\hline FIGO Stage & & & & & & & $\begin{array}{l}8.724 \\
\left(0.003^{\star}\right)\end{array}$ \\
\hline$H$ & 39 & 7 & 10 & 11 & 11 & 56.41 & \\
\hline III-IV & 47 & 2 & 5 & 22 & 18 & 85.11 & \\
\hline Differentiation & & & & & & & $\begin{array}{l}1.939 \\
(0.164)\end{array}$ \\
\hline Well-moderately & 47 & 7 & 9 & 21 & 10 & 65.96 & \\
\hline Poorly-undifferentiated & 39 & 2 & 6 & 12 & 19 & 79.48 & \\
\hline Lymph node metastasis & & & & & & & $\begin{array}{l}1.418 \\
(0.492)\end{array}$ \\
\hline No & 57 & 6 & 12 & 19 & 20 & 68.42 & \\
\hline Yes & 22 & 2 & 2 & 10 & 8 & 81.82 & \\
\hline No lymphadenectomy & 7 & 1 & 1 & 4 & 1 & 71.43 & \\
\hline Pathological subtype & & & & & & & $\begin{array}{l}7.963 \\
(0.093)\end{array}$ \\
\hline Mucinous & 6 & 2 & 2 & 2 & 0 & 33.33 & \\
\hline Endometrioid & 7 & 1 & 2 & 3 & 1 & 57.14 & \\
\hline Serous & 57 & 5 & 10 & 23 & 19 & 73.68 & \\
\hline $\begin{array}{l}\text { Poorly differentiated } \\
\text { adenocarcinoma }\end{array}$ & 10 & 1 & 1 & 3 & 5 & 80.00 & \\
\hline Clear cell carcinoma & 6 & 0 & 0 & 2 & 4 & 100.00 & \\
\hline
\end{tabular}


Table 3

Correlation between UBE2T expression and clinicopathological features in ovarian cancer patients of the GSE73614 dataset

\begin{tabular}{|c|c|c|c|c|c|}
\hline Characteristics & $\begin{array}{l}\text { Cases } \\
(n=107)\end{array}$ & & pression & $\begin{array}{l}\text { High positive rate } \\
\text { (\%) }\end{array}$ & $\begin{array}{l}\text { Chi square Value } \\
\text { ( } P \text { value })\end{array}$ \\
\hline \multicolumn{5}{|l|}{ Age (years) } & $0.231(0.631)$ \\
\hline$₫ 60$ & 52 & 27 & 25 & 48.08 & \\
\hline$\geq 60$ & 55 & 26 & 29 & 52.73 & \\
\hline \multicolumn{5}{|l|}{ FIGO Stage } & $5.856\left(0.016^{\star}\right)$ \\
\hline III & 48 & 30 & 18 & 37.50 & \\
\hline IIII-IV & 59 & 23 & 36 & 61.02 & \\
\hline \multicolumn{5}{|l|}{ Differentiation } & $3.645(0.162)$ \\
\hline Moderately & 29 & 17 & 12 & 41.37 & \\
\hline Poorly & 69 & 34 & 35 & 50.72 & \\
\hline Undifferentiated & 9 & 2 & 7 & 77.78 & \\
\hline \multicolumn{5}{|l|}{ Pathological subtype } & $14.772\left(0.001^{\star}\right)$ \\
\hline Clear cell carcinoma & 37 & 27 & 10 & 27.03 & \\
\hline Endometrioid & 66 & 26 & 40 & 60.61 & \\
\hline Serous & 4 & 0 & 4 & 100 & \\
\hline \multicolumn{6}{|c|}{ Relationship between UBE2T expression and prognosis in patients with epithelial ovarian cancer } \\
\hline \multicolumn{6}{|c|}{ 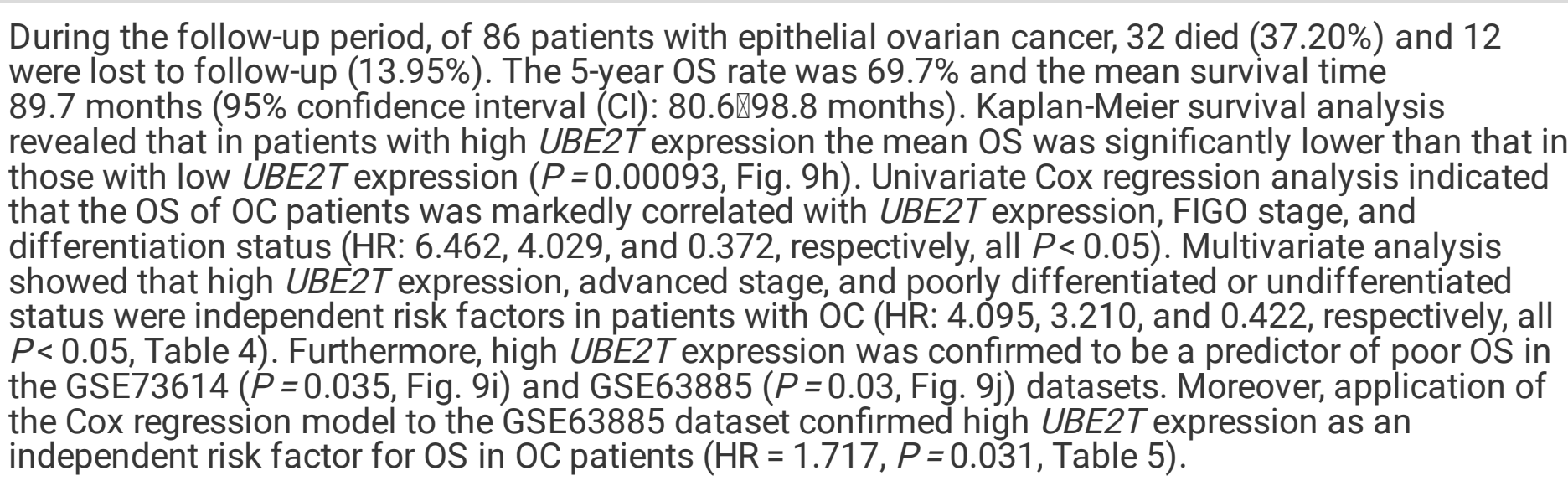 } \\
\hline
\end{tabular}


Table 4

Univariate and multivariate analyses of overall survival in ovarian cancer clinical samples

\begin{tabular}{|c|c|c|c|c|}
\hline Variable & $\begin{array}{l}\text { Univariate } \\
\text { analysis }\end{array}$ & & $\begin{array}{l}\text { Multivariate } \\
\text { analysis }\end{array}$ & \\
\hline & $\mathrm{HR}(95 \% \mathrm{Cl})$ & $\begin{array}{l}P \\
\text { value }\end{array}$ & $\mathrm{HR}(95 \% \mathrm{Cl})$ & $\begin{array}{l}P \\
\text { value }\end{array}$ \\
\hline \multicolumn{5}{|l|}{ Age } \\
\hline$₫ 60$ vs. $\geq 60$ & $\begin{array}{l}1.345(0.604- \\
2.995)\end{array}$ & 0.469 & & \\
\hline \multicolumn{5}{|l|}{ UBE2T expression } \\
\hline High vs. low & $\begin{array}{l}6.462(1.875- \\
22.266)\end{array}$ & 0.003 & $\begin{array}{l}4.095(1.154- \\
14.533)\end{array}$ & 0.029 \\
\hline \multicolumn{5}{|l|}{ FIGO Stage } \\
\hline I-II vs. III-IV & $\begin{array}{l}4.029(1.733- \\
9.367)\end{array}$ & 0.001 & $\begin{array}{l}3.210(1.348- \\
7.640)\end{array}$ & 0.008 \\
\hline \multicolumn{5}{|l|}{ Differentiation } \\
\hline $\begin{array}{l}\text { Poorly-undifferentiated vs. well- } \\
\text { moderately }\end{array}$ & $\begin{array}{l}0.372(0.179- \\
0.772)\end{array}$ & 0.008 & $\begin{array}{l}0.422(0.201- \\
0.884)\end{array}$ & 0.022 \\
\hline \multicolumn{5}{|l|}{ Pathological subtype } \\
\hline Serous vs. nonserous & $\begin{array}{l}1.177(0.575- \\
2.409)\end{array}$ & 0.656 & & \\
\hline \multicolumn{5}{|l|}{ Lymph node metastasis } \\
\hline Yes vs. no & $\begin{array}{l}1.964(0.959- \\
4.022)\end{array}$ & 0.065 & & \\
\hline
\end{tabular}


Table 5

Univariate and multivariate analyses of overall survival in ovarian cancer patients of the GSE63885 dataset

\begin{tabular}{|c|c|c|c|c|}
\hline \multirow[t]{2}{*}{ Variable } & \multicolumn{2}{|c|}{ Univariate analysis } & \multicolumn{2}{|l|}{$\begin{array}{l}\text { Multivariate } \\
\text { analysis }\end{array}$} \\
\hline & $\mathrm{HR}(95 \% \mathrm{Cl})$ & $\begin{array}{l}P \\
\text { value }\end{array}$ & $\mathrm{HR}(95 \% \mathrm{Cl})$ & $\begin{array}{l}P \\
\text { value }\end{array}$ \\
\hline \multicolumn{5}{|l|}{ UBE2T expression } \\
\hline High vs. low & $\begin{array}{l}1.711(1.048- \\
2.793)\end{array}$ & 0.032 & $\begin{array}{l}1.717(1.052- \\
2.803)\end{array}$ & 0.031 \\
\hline \multicolumn{5}{|l|}{ FIGO Stage } \\
\hline II-III vs. IV & $\begin{array}{l}2.214(1.093- \\
4.485)\end{array}$ & 0.027 & $\begin{array}{l}2.226(1.102- \\
4.495)\end{array}$ & 0.026 \\
\hline \multicolumn{5}{|l|}{ Differentiation } \\
\hline $\begin{array}{l}\text { Poorly-undifferentiated vs. } \\
\text { moderately }\end{array}$ & $\begin{array}{l}2.321(1.040- \\
5.178)\end{array}$ & 0.04 & & \\
\hline Pathological subtype & $\begin{array}{l}1.293(0.517- \\
3.233)\end{array}$ & 0.582 & & \\
\hline
\end{tabular}

\section{Discussion}

E2-conjugating enzymes play a central role in the formation and progression of various tumors[30]. Although the role of some UBE2 members in OC has been confirmed, to our knowledge, no studies have comprehensively analyzed the prognostic value and functional mechanism of the UBE2 family in OC. In this study, a systematic bioinformatics analysis was conducted to evaluate the potential role of UBE2 family genes as biomarkers of OC.

$U B E 2 A$ and $U B E 2 B$ are two RAD6 homologs originally identified in yeast and required for DNA repair[31]. They are both upregulated in $\mathrm{OC}$ compared to normal ovarian tissues, and their high expression is associated with poor prognosis in OC patients[6]. Downregulation of RAD6 was previously found to reduce the expression of the cancer stem cell markers, $A L D H 1 A 1$ and $S O X 2$, and to increase the sensitivity of $O C$ cells to carboplatin[32]. UBE2C is a key regulator of cell cycle progression and mitosis[33]. It is highly expressed in epithelial ovarian cancer (EOC), especially in high-grade serous adenocarcinoma, and its high expression is an independent risk factor affecting the prognosis of EOC patients[34]. Another study showed that blocking UBE2C expression by RNA interference inhibits the growth of OC cell lines[35]. Two neddylation conjugating E2 enzymes, UBE2M and UBE2F, were found to play essential roles in paclitaxel (PTX)-induced cytotoxicity and tubulin polymerization in OC cell lines. A recent study reported that $U B E 2 M$ and $U B E 2 F$ knockdown impairs protein neddylation and reduces the antitumor 
activity of PTX in OC, suggesting new research directions on PTX chemoresistance [36]. The results of our bioinformatics analyses were consistent with previous reports. Kaplan-Meier plotter analysis showed that increased UBE2A and UBE2B expression was associated with poor PFS in OC patients and poor OS in patients with serous ovarian cancer. UBE2C expression was significantly higher in OC compared to normal tissues, and predicted poor prognosis. Although UBE2F and UBE2M were significantly more expressed in $\mathrm{OC}$ compared to normal tissues, their levels had no correlation with prognosis.

UBE2/ and UBE2N are involved in DNA repair and cell apoptosis[37, 38]. UBE2/ expression was higher in $\mathrm{OC}$ than in normal tissues, and correlated with clinicopathologic parameters. Moreover, UBE2/ expression level was higher in ovarian serous carcinoma compared to ovarian myxoid carcinoma, endometrioid carcinoma, and clear cell carcinoma, and in advanced or poorly differentiated EOC compared to early or well-differentiated EOC. Guo et al. reached similar conclusions, suggesting that UBE2/ may be involved in the progression and histopathological differentiation of $O C[39,40]$. The downregulation of UBE2N in OC may be related to the acquisition of resistance to PTX through the DNMT1-CHFR-Aurora A pathway. Thus, $U B E 2 N$ is a potential target of drugs aiming at reversing PTX resistance in patients with OC[41]. UBE2R2 plays a role in the ubiquitin-proteasome pathway[42], and the combination of UBE2R2-SMYD3-p53 significantly promotes p53 ubiquitination and degradation, thereby inducing EOC progression and metastasis[43]. However, some of our data were inconsistent with previous results. Oncomine analysis showed that UBE2I and UBE2N were significantly downregulated and upregulated in OC, respectively. $U B E 2 N$ overexpression was associated with poor PFS in OC patients, while UBE2R2 downregulation correlated with poor OS and PFS in patients with ovarian serous tumors. We attribute these contradictory findings to background heterogeneity between different databases, as well as to the limited number of samples.

Currently, little is known about the biological function of UBE2G, and even less about its role in tumors. Previous studies found that $U B E 2 G$ is strongly expressed in the skeletal muscle and participates in the degradation of muscle-specific proteins[44]. Our findings demonstrated that the expression level of UBE2G in OC was significantly lower than in normal ovarian tissues, and that this downregulation was associated with poor PFS in OC patients, suggesting a tumor-suppressive role of this protein in OC. This possibility needs to be verified by further experiments.

Although there are few studies on the roles of UBE2S and UBE2V2 in OC, both genes are involved in the occurrence and progression of other tumors. UBE2S was reported to be aberrantly expressed in some gynecological tumors, including breast, cervical, and endometrial cancer, and its knockdown inhibits the proliferation of cancer cells and promotes apoptosis[45-47]. UBE2V2 overexpression seems to be involved in the pathogenesis of gastric cancer, and is significantly associated with poor prognosis in ERpositive/HER2-negative breast cancer[48, 49]. The current study demonstrated that both UBE2V2 and $U B E 2 S$ were significantly upregulated in $\mathrm{OC}$ compared to normal tissues. However, there was no obvious correlation between their expression levels and prognosis. 
UBE2T, also known as FANCT or HSPC150, is essential for FANCD2 monoubiquitination. It combines with a specific ubiquitin E3 ligase to degrade specific substrates, contributing to DNA repair in the Fanconi anemia pathway and playing a key role in cell proliferation and the maintenance of genomic stability[50]. Recently, UBE2T expression has been reported to be significantly upregulated in hepatocellular carcinoma[10], renal cell carcinoma[51], prostate cancer[15], breast cancer, and lung adenocarcinoma[52], and its high expression is associated with poor prognosis in patients. Consistently, after integrating the data on gene expression and prognostic value obtained from different databases, we found that UBE2T was significantly overexpressed in $\mathrm{OC}$ compared to normal ovarian tissues, and that its high expression was associated with poor prognosis in both $\mathrm{OC}$ and serous ovarian cancer.

Various studies have shown that specific genetic alterations are related to cancer prognosis and potential predictors of cancer metastasis[53,54]. To verify the correlation between alterations in UBE2 genes and $\mathrm{OC}$, the $\mathrm{CBioPortal}$ database was analyzed, revealing an overall rate of genetic alterations ranging from $13.5-28.47 \%$ in the $\mathrm{OC}$ dataset, and a percentage of individual gene alterations ranging from $0.9-4 \%$. However, alterations in single UBE2 genes had no significant impact on OS or DFS, suggesting that these changes did not directly affect $\mathrm{OC}$ prognosis.

Since UBE2 $T$ displayed the greatest correlation with OC among UBE2 family members, its clinical effects in $\mathrm{OC}$ were further investigated. IHC staining and GEO database analysis confirmed that UBE2T expression was significantly increased in ovarian epithelial malignant tumors compared to ovarian epithelial borderline tumors, benign tumor, and normal tissues. The expression level of UBE2T in FIGO stage III-IV tumors was significantly higher than in FIGO stage I-II tumors, suggesting that UBE2T may promote $\mathrm{OC}$ progression. In addition, Cox multivariate analysis indicated high UBE2T expression as an independent risk factor affecting the prognosis of patients with EOC. Therefore, UBE2T is a candidate biomarker for the prediction of OC prognosis.

Multiple evidence indicates that UBE2T acts as an oncogene in a variety of tumors, but its molecular mechanism of action varies in different types of cancer. One study showed that UBE2T plays a role in the proliferation and invasion of hepatocellular carcinoma cells by regulating the $G 2 / M$ transition of the cell cycle through the cyclin B1-CDK1 pathway[55], while another study found that UBE2T enhances p53 ubiquitination and degradation, promoting the carcinogenesis of hepatocellular carcinoma[10]. Furthermore, UBE2T downregulation reduces the activity of the PI3K/Akt signaling pathway, thus inhibiting the proliferation and migration of renal cell carcinoma [51] and osteosarcoma cells[56]. Moreover, UBE2T promotes breast cancer progression by directly regulating the BRCA1/BARD1 complex[12]. Furthermore, UBE2T promotes nasopharyngeal carcinoma cell proliferation, invasion, and metastasis by activating the AKT/GSK3 $\beta / \beta$-catenin pathway[57]. Finally, UBE2T silencing in bladder or gastric cancer was found to induce cell cycle arrest in $\mathrm{G} 2 / \mathrm{M}$ phase, thereby promoting cancer cell apoptosis and inhibiting tumor growth $[13,14]$. However, the role of UBE2T in OC has not yet been investigated. 
To clarify the role of UBE2T in OC at the molecular level, the functions and relevant pathways of UBE2T co-expressed genes were investigated by enrichment analysis using the Metascape database. These genes were mainly involved in processes related to cell cycle, including cell division, cell cycle phase transition, DNA replication, and DNA repair, and predominantly implicated in cell cycle regulation, Fanconi anemia signaling, and p53 signaling. GSEA results revealed that patients with high UBE2C expression were significantly enriched in E2F_TARGETS and G2M_ CHECKPOINT. KEGG pathway analysis elucidated that the UBE2T high expression mutation group was significantly enriched in focal adhesion, calcium signaling, and ECM-receptor interactions. These processes are critical for tumor progression, which could explain the role and molecular function of UBE2T in OC.

In patients with high UBE2T expression, TP53, TTN, DST, FAT3, CSMD1, MUC16, and MUC17 exhibited a significant rate of alterations. TP53 mutation frequency in ovarian serous carcinomas has been reported to range from $50 \%$ and $80 \%[58]$; ubiquitous TP53 mutations are characteristic of high-grade serous ovarian cancer (HGSOC) and related to relapse[59]. TTN encodes a large polypeptide expressed in many cancer cell types and involved in oncogenesis, and its mutation has been found in $\mathrm{OC}$ by using an the next-generation sequencing (NGS) library[60]. CSDM1 was present in the genome-wide homozygous deletion profiling of HGSOC[61]. Gene expression profiling showed that MUC16 and MUC17 are overexpressed in the majority of serous and mucinous ovarian carcinomas, respectively[62, 63]. We hypothesize that mutations in these genes may be related to the high level of UBE2T expression in OC. In addition, gene amplification and hypomethylation were also identified as possible causes of UBE2T overexpression.

Growing evidence is being provided that the interaction between immune and cancer cells in the tumor microenvironment has an impact on tumor progression[30]. OC is an immunogenic tumor that can be recognized and attacked by the immune system. The accumulation of TILs in OC microenvironment is related to prolonged OS of the patients, while immune escape events are associated with poor prognosis $[64,65]$. In this study, the correlation between UBE2T expression and the levels of infiltrating immune cells was analyzed by using the TISIDB-OV database. Particularly strong correlations were found between UBE2T expression and the level of specific TIL populations (eosinophils, Act_CD4, neutrophils, and memory B cells), immune inhibitors (CSF1R, TGFB1, KDR, and CD160), immune stimulators (NTSE, TNFSF14, and TNFSF15), and MHC molecules (HLA-E, HLA-DOA, HLA-DQA1, and HLA-DQB1). The infiltration of $B$ cells in HGSOC promotes anti-tumor responses, and chemotherapy enhances memory $B$ cell response. Hence, the presence of B cells is a predictor of improved survival in patients with OC[66]. $C S F 1 R$ is overexpressed in $O C$ and the determination of its circulating levels proved useful for disease detection and the evaluation of therapeutic efficacy[67]. It has been reported that the cells of ovarian carcinoma produce large amounts of TGF- $\beta 1$, which facilitates their escape from the immune system, and the clinical efficacy of immunotherapy based on TGF- $\beta 1$-silenced tumor vaccines for OC has been investigated[68]. We speculate that UBE2T overexpression may cause the reduction or depletion of immune cells, leading to tumor progression by immune escape. 


\section{Conclusions}

In summary, our bioinformatics analyses highlighted a potential role of UBE2s in OC onset and progression. However, our findings need to be supported by additional research and clinical data. $\mathrm{IHC}$ and GEO analysis confirmed that UBE2T was significantly upregulated in $O C$, and that its high expression was an independent risk factor and prognostic predictor for OC. UBE2 $T$ is also a potential biomarker for early diagnosis and a candidate immune-related therapeutic target for OC. However, the biological properties of $U B E 2 T$ and the molecular events related to its overexpression in $\mathrm{OC}$ need to be further explored.

\section{Abbreviations}

OC

Ovarian cancer; EOC:Epithelial ovarian cancer; GEPIA:The Gene Expression Profiling Interactive Analysis; TCGA:The Cancer Genome Atlas; GTEx:Genotype-tissue Expression dataset projects; GEO:Gene Expression Omnibus; OS:Overall survial; DFS:Disease-free survival; PFS:Progression-free survival; PPI:Protein-protein interaction; GO:Gene Ontology; KEGG:Kyoto Encyclopedia of Genes and Genomes; TILs:Tumor-infiltrating lymphocytes; MHC:Major histocompatibility complex; FIGO:International Federation of Gynecology and Obstetrics; Cl:Confidence interval; UBE2:Ubiquitin conjugating enzyme E2; GSEA:Gene set enrichment analysis; CNV:Copy number variation; IHC:Immunohistochemical; HR:Hazard ratio; OCCs:Ovarian cancer cells; HGSOC:High-grade serous ovarian cancer.

\section{Declarations}

\section{Ethics approval and consent to participate}

Ethical approval for this study was obtained from the Clinical Research Ethics Committee of Shengjing Hospital of China Medical University (Approval Number: 2020PS533K), and all patients provided signed informed consent in accordance with the Declaration of Helsinki.

\section{Consent for publication}

\section{All authors have agreed to publish this manuscript}

\section{Availability of data and materials}

The data generated and/or analysed in this study are available from GEO database (https://www.ncbi.nlm.nih.gov/geo), TCGA (https://portal.gdc.cancer.gov/), cBioPortal (http://www.cbioportal.org/), and Xena (https://xenabrowser.net/datapages/).

\section{Competing interests}


The authors declare that they have no conflict of interest.

\section{Funding}

This work was supported by grants from National Natural Science Foundation of China Youth Science Foundation (No. 81602438), Doctoral start-up fund of Liaoning Province (Grant No. 201601133), 345 Talent Project of Shengjing Hospital.

\section{Authors' Contributions}

LZ and RZ designed research. RZ and HX wrote manuscript. FL performed the immunohistochemical assay. SW involved in data collection and data statistical analysis. LZ provided fund support and critically reviewed the manuscript. All authors read and approved the final manuscript.

\section{Acknowledgements}

None.

\section{References}

1. Siegel RL, Miller KD, Jemal A. Cancer statistics, 2018. CA Cancer J Clin. 2018;68(1):7.

2. Rottmann M, Burges A, Mahner S, Anthuber C, Beck T, Grab D, et al. Cancer of the ovary, fallopian tube, and peritoneum: a population-based comparison of the prognostic factors and outcomes. $J$ Cancer Res Clin Oncol. 2017;143(9):1833-44.

3. Jadidi-Niaragh F, Ghalamfarsa G, Yousefi M, Tabrizi MH, Shokri F. Regulatory T cells in chronic lymphocytic leukemia: implication for immunotherapeutic interventions. Tumour Biol. 2013;34(4):2031-9.

4. Clague MJ, Heride C, Urbé S. The demographics of the ubiquitin system. Trends Cell Biol. 2015;25(7):417-26.

5. Garg P, Ceccarelli DF, Keszei AFA, Kurinov I, Sicheri F, Sidhu SS. Structural and Functional Analysis of Ubiquitin-based Inhibitors That Target the Backsides of E2 Enzymes. J Mol Biol. 2020;432:952-66.

6. Somasagara RR, Tripathi K, Spencer SM, Clark DW, Barnett R, Bachaboina L, et al. Rad6 upregulation promotes stem cell-like characteristics and platinum resistance in ovarian cancer. Biochem Biophys Res Commun. 2016;469(3):449-55.

7. Matsumoto A, Ishibashi Y, Urashima M, Omura N, Nakada K, Nishikawa K, et al. High UBCH10 protein expression as a marker of poor prognosis in esophageal squamous cell carcinoma. Anticancer Res. 2014;34(2):955-61.

8. Zhou W, Xu J, Li H, Xu M, Chen ZJ, Wei W, et al. Neddylation E2 UBE2F Promotes the Survival of Lung Cancer Cells by Activating CRL 5 to Degrade NOXA via the K11 Linkage. Clin Cancer Res. 
2017;23(4):1104-16.

9. Machida YJ, Machida Y, Chen Y, Gurtan AM, Kupfer GM, D'Andrea AD, et al. UBE2T is the E2 in the Fanconi anemia pathway and undergoes negative autoregulation. Mol Cell. 2006;23(4):589-96.

10. Liu LP, Yang M, Peng QZ, Li MY, Zhang YS, Guo YH, et al. UBE2T promotes hepatocellular carcinoma cell growth via ubiquitination of p53. Biochem Biophys Res Commun. 2017;493(1):20-7.

11. Hao J, Xu A, Xie X, Hao J, Tian T, Gao S, et al. Elevated expression of UBE2T in lung cancer tumors and cell lines. Tumour Biol. 2008;29(3):195-203.

12. Ueki T, Park JH, Nishidate T, Kijima K, Hirata K, Nakamura Y, et al. Ubiquitination and downregulation of BRCA1 by ubiquitin-conjugating enzyme E2T overexpression in human breast cancer cells. Cancer Res. 2009;69(22):8752-60.

13. Luo C, Yao Y, Yu Z, Zhou H, Guo L, Zhang J, et al. UBE2T knockdown inhibits gastric cancer progression. Oncotarget. 2017;8(20):32639-54.

14. Gong YQ, Peng D, Ning XH, Yang XY, Li XS, Zhou LQ, et al. UBE2T silencing suppresses proliferation and induces cell cycle arrest and apoptosis in bladder cancer cells. Oncol Lett. 2016;12(6):4485-92.

15. Wen M, Kwon Y, Wang Y, Mao JH, Wei G. Elevated expression of UBE2T exhibits oncogenic properties in human prostate cancer. Oncotarget. 2015;6(28):25226-39.

16. Rhodes DR, Yu J, Shanker K, Deshpande N, Varambally R, Ghosh D, et al. ONCOMINE: a cancer microarray database and integrated data-mining platform. Neoplasia. 2004;6(1):1-6.

17. Tang Z, Li C, Kang B, Gao G, Li C, Zhang Z. GEPIA: a web server for cancer and normal gene expression profiling and interactive analyses. Nucleic Acids Res. 2017;45:W98-102.

18. Karlan BY, Dering J, Walsh C, Orsulic S, Lester J, Anderson LA, et al. POSTN/TGFBI-associated stromal signature predicts poor prognosis in serous epithelial ovarian cancer. Gynecol Oncol. 2014;132(2):334-42.

19. Winterhoff B, Hamidi H, Wang C, Kalli KR, Fridley BL, Dering J, et al. Molecular classification of high grade endometrioid and clear cell ovarian cancer using TCGA gene expression signatures. Gynecol Oncol. 2016;141(1):95-100.

20. Lisowska KM, Olbryt M, Student S, Kujawa KA, Cortez AJ, Simek K, et al. Unsupervised analysis reveals two molecular subgroups of serous ovarian cancer with distinct gene expression profiles and survival. J Cancer Res Clin Oncol. 2016;142(6):1239-52.

21. Gyorffy B, Lánczky A, Szállási Z. Implementing an online tool for genome-wide validation of survivalassociated biomarkers in ovarian-cancer using microarray data from 1287 patients. Endocr Relat Cancer. 2012;19(2):197-208.

22. Szklarczyk D, Morris JH, Cook H, Kuhn M, Wyder S, Simonovic M, et al. The STRING database in 2017: quality-controlled protein-protein association networks, made broadly accessible. Nucleic Acids Res. 2017;45:D362-8.

23. Zhou Y, Zhou B, Pache L, Chang M, Khodabakhshi AH, Tanaseichuk O, et al. Metascape provides a biologist-oriented resource for the analysis of systems-level datasets. Nat Commun. 
2019;10(1):1523.

24. Ru B, Wong CN, Tong Y, Zhong JY, Zhong SSW, Wu WC, et al. TISIDB: an integrated repository portal for tumor-immune system interactions. Bioinformatics. 2019;35(20):4200-02.

25. Yoshihara K, Tajima A, Komata D, Yamamoto T, Kodama S, Fujiwara H, et al. Gene expression profiling of advanced-stage serous ovarian cancers distinguishes novel subclasses and implicates ZEB2 in tumor progression and prognosis. Cancer Sci. 2009;100(8):1421-8.

26. Lu KH, Patterson AP, Wang L, Marquez RT, Atkinson EN, Baggerly KA, et al. Selection of potential markers for epithelial ovarian cancer with gene expression arrays and recursive descent partition analysis. Clin Cancer Res. 2004;10(10):3291-300.

27. Adib TR, Henderson S, Perrett C, Hewitt D, Bourmpoulia D, Ledermann J, et al. Predicting biomarkers for ovarian cancer using gene-expression microarrays. Br J Cancer. 2004;90(3):686-92.

28. Welsh JB, Zarrinkar PP, Sapinoso LM, Kern SG, Behling CA, Monk BJ, et al. Analysis of gene expression profiles in normal and neoplastic ovarian tissue samples identifies candidate molecular markers of epithelial ovarian cancer. Proc Natl Acad Sci USA. 2001;98(3):1176-81.

29. Bonome T, Levine DA, Shih J, Randonovich M, Pise-Masison CA, Bogomolniy F, et al. A gene signature predicting for survival in suboptimally debulked patients with ovarian cancer. Cancer Res. 2008;68(13):5478-86.

30. Hosseini SM, Okoye I, Chaleshtari MG, Hazhirkarzar B, Mohamadnejad J, Azizi G, et al. E2 ubiquitinconjugating enzymes in cancer: Implications for immunotherapeutic interventions. Clin Chim Acta. 2019;498:126-34.

31. Koken MH, Reynolds P, Jaspers-Dekker I, Prakash L, Prakash S, Bootsma D, et al. Structural and functional conservation of two human homologs of the yeast DNA repair gene RAD6. Proc Natl Acad Sci USA. 1991;88(20):8865-9.

32. Somasagara RR, Spencer SM, Tripathi K, Clark DW, Mani C, Madeira da Silva L, et al. RAD6 promotes DNA repair and stem cell signaling in ovarian cancer and is a promising therapeutic target to prevent and treat acquired chemoresistance. Oncogene. 2017;36(48):6680-90.

33. Zhang J, Liu X, Yu G, Liu L, Wang J, Chen X, et al. UBE2C Is a Potential Biomarker of Intestinal-Type Gastric Cancer With Chromosomal Instability. Front Pharmacol. 2018;9:847.

34. Gong Y, Wang D, Lin L, Dai J, Yu L. The expression of ubiquitin-conjugating enzyme E2C and KAl1 in ovarian carcinoma and their clinical significance. Medicine. 2019;98(46):e17896.

35. Berlingieri MT, Pallante P, Guida M, Nappi C, Masciullo V, Scambia G, et al. UbcH10 expression may be a useful tool in the prognosis of ovarian carcinomas. Oncogene. 2007;26(14):2136-40.

36. Hong X, Li S, Li W, Xie M, Wei Z, Guo H, et al. Disruption of protein neddylation with MLN4924 attenuates paclitaxel-induced apoptosis and microtubule polymerization in ovarian cancer cells. Biochem Biophys Res Commun. 2019;508(3):986-90.

37. Zhang M, Windheim M, Roe SM, Peggie M, Cohen P, Prodromou C, et al. Chaperoned ubiquitylationcrystal structures of the CHIP U box E3 ubiquitin ligase and a CHIP-Ubc13-Uev1a complex. Mol Cell. 2005;20(4):525-38. 
38. Jackson SP, Durocher D. Regulation of DNA damage responses by ubiquitin and SUMO. Mol Cell. 2013;49(5):795-807.

39. Zhu H, Yu JJ. Gene expression patterns in the histopathological classification of epithelial ovarian cancer. Exp Ther Med. 2010;1(1):187-92.

40. Guo Y, Fu P, Zhu H, Reed E, Remick SC, Petros W, et al. Correlations among ERCC1, XPB, UBE2I, EGF, TAL2 and ILF3 revealed by gene signatures of histological subtypes of patients with epithelial ovarian cancer. Oncol Rep. 2012;27(1):286-92.

41. Zhang $X$, Feng $Y$, Wang $X Y$, Zhang YN, Yuan CN, Zhang SF, et al. The inhibition of UBC13 expression and blockage of the DNMT1-CHFR-Aurora A pathway contribute to paclitaxel resistance in ovarian cancer. Cell Death Dis. 2018;9(2):93.

42. Xu W, Hu GQ, Da Costa C, Tang JH, Li QR, Du L, et al. Long noncoding RNA UBE2R2-AS1 promotes glioma cell apoptosis via targeting the miR-877-3p/TLR4 axis. Onco Targets Ther. 2019;12:3467-80.

43. Zhang L, Jin Y, Yang H, Li Y, Wang C, Shi Y, et al. SMYD3 promotes epithelial ovarian cancer metastasis by down-regulating p53 protein stability and promoting p53 ubiquitination. Carcinogenesis. 2019;40:1492-503.

44. Watanabe TK, Kawai A, Fujiwara T, Maekawa H, Hirai Y, Nakamura Y, et al. Molecular cloning of UBE2G, encoding a human skeletal muscle-specific ubiquitin-conjugating enzyme homologous to UBC7 of C. elegans. Cytogenet Cell Genet. 1996;74:146-8.

45. Ayesha AK, Hyodo T, Asano E, Sato N, Mansour MA, Ito S, et al. UBE2S is associated with malignant characteristics of breast cancer cells. Tumour Biol. 2016;37(1):763-72.

46. Lin TH, Hsu WH, Tsai PH, Huang YT, Lin CW, Chen KC, et al. Dietary flavonoids, luteolin and quercetin, inhibit invasion of cervical cancer by reduction of UBE2S through epithelial-mesenchymal transition signaling. Food Funct. 2017;8(4):1558-68.

47. Lin M, Lei T, Zheng J, Chen S, Du L, Xie H. UBE2S mediates tumor progression via SOX6/ $\beta$-Catenin signaling in endometrial cancer. Int J Biochem Cell Biol. 2019;109:17-22.

48. Cheng L, Wang P, Yang S, Yang Y, Zhang Q, Zhang W, et al. Identification of genes with a correlation between copy number and expression in gastric cancer. BMC Med Genomics. 2012;5:14.

49. Santarpia L, Iwamoto T, Di Leo A, Hayashi N, Bottai G, Stampfer M, et al. DNA Repair Gene Patterns as Prognostic and Predictive Factors in Molecular Breast Cancer Subtypes. Oncologist. 2013;18(10):1063-73.

50. Alpi AF, Chaugule V, Walden H. Mechanism and disease association of E2-conjugating enzymes: lessons from UBE2T and UBE2L3. Biochem J. 2016;473(20):401-19.

51. Hao P, Kang B, Li Y, Hao W, Ma F. UBE2T promotes proliferation and regulates PI3K/Akt signaling in renal cell carcinoma. Mol Med Rep. 2019;20(2):1212-20.

52. Perez-Peña J, Corrales-Sánchez V, Amir E, Pandiella A, Ocana A. Ubiquitin-conjugating enzyme E2T (UBE2T) and denticleless protein homolog (DTL) are linked to poor outcome in breast and lung cancers. Sci Rep. 2017;7(1):17530. 
53. Hieronymus H, Murali R, Tin A, Yadav K, Abida W, Moller H, et al. Tumor copy number alteration burden is a pan-cancer prognostic factor associated with recurrence and death. Elife. 2018;7.

54. Pearlman A, Upadhyay K, Cole K, Loke J, Sun K, Fineberg S, et al. Robust genomic copy number predictor of pan cancer metastasis. Genes Cancer. 2018;9:66-77.

55. Liu LL, Zhu JM, Yu XN, Zhu HR, Shi X, Bilegsaikhan E, et al. UBE2T promotes proliferation via G2/M checkpoint in hepatocellular carcinoma. Cancer Manag Res. 2019;11:8359-70.

56. Wang $Y$, Leng $H$, Chen $H$, Wang L, Jiang N, Huo X, et al. Knockdown of UBE2T Inhibits Osteosarcoma Cell Proliferation, Migration, and Invasion by Suppressing the PI3K/Akt Signaling Pathway. Oncol Res. 2016;24(5):361-9.

57. Hu W, Xiao L, Cao C, Hua S, Wu D. UBE2T promotes nasopharyngeal carcinoma cell proliferation, invasion, and metastasis by activating the AKT/GSK3 $\beta / \beta$-catenin pathway. Oncotarget. 2016;7(12):15161-72.

58. Salani R, Kurman RJ, Giuntoli R, Gardner G, Bristow R, Wang TL, et al. Assessment of TP53 mutation using purified tissue samples of ovarian serous carcinomas reveals a higher mutation rate than previously reported and does not correlate with drug resistance. Int J Gynecol Cancer. 2008;18(3):487-91.

59. Nelson L, Tighe A, Golder A, Littler S, Bakker B, Moralli D, et al. living biobank of ovarian cancer ex vivo models reveals profound mitotic heterogeneity. Nat Commun. 2020;11(1):822.

60. Zhang L, Luo M, Yang H, Zhu S, Cheng X, Qing C. Next-generation sequencing-based genomic profiling analysis reveals novel mutations for clinical diagnosis in Chinese primary epithelial ovarian cancer patients. J Ovarian Res. 2019;12(1):19.

61. Kuo KT, Guan B, Feng Y, Mao TL, Chen X, Jinawath N, et al. Analysis of DNA Copy Number Alterations in Ovarian Serous Tumors Identifies New Molecular Genetic Changes in Low-Grade and High-Grade Carcinomas. Cancer Res. 2009;69(9):4036-42.

62. Comamala $M$, Pinard $M$, Thériault $C$, Matte I, Albert $A$, Boivin $M$, et al. Downregulation of cell surface CA125/MUC16 induces epithelial-to-mesenchymal transition and restores EGFR signalling in NIH:OVCAR3 ovarian carcinoma cells. Br J Cancer. 2011;104(6):989-99.

63. Yang CW, Chang CY, Lai MT, Chang HW, Lu CC, Chen Y, et al. Genetic variations of MUC17 are associated with endometriosis development and related infertility. BMC Med Genet. 2015;16:60.

64. Yang S, Yin X, Yue Y, Wang S. Application Of Adoptive Immunotherapy In Ovarian Cancer. Onco Targets Ther. 2019;12:7975-91.

65. Ghisoni E, Imbimbo M, Zimmermann S, Valabrega G. Ovarian Cancer Immunotherapy: Turning up the Heat. Int J Mol Sci. 2019;20(12).

66. Gupta P, Chen C, Chaluvally-Raghavan P, Pradeep S. B Cells as an Immune-Regulatory Signature in Ovarian Cancer. Cancers (Basel). 2019;11(7).

67. Farag AK, Hassan AHE, Ahn BS, Park KD, Roh EJ. Reprofiling of pyrimidine-based DAPK1/CSF1R dual inhibitors: identification of 2,5-diamino-4-pyrimidinol derivatives as novel potential anticancer lead compounds. J Enzyme Inhib Med Chem. 2020;35(1):311-24. 
68. Wei H, Liu P, Swisher E, Yip YY, Tse JH, Agnew K, et al. Silencing of the TGF- $\beta 1$ gene increases the immunogenicity of cells from human ovarian carcinoma. J Immunother. 2012;35(3):267-75.

Figures
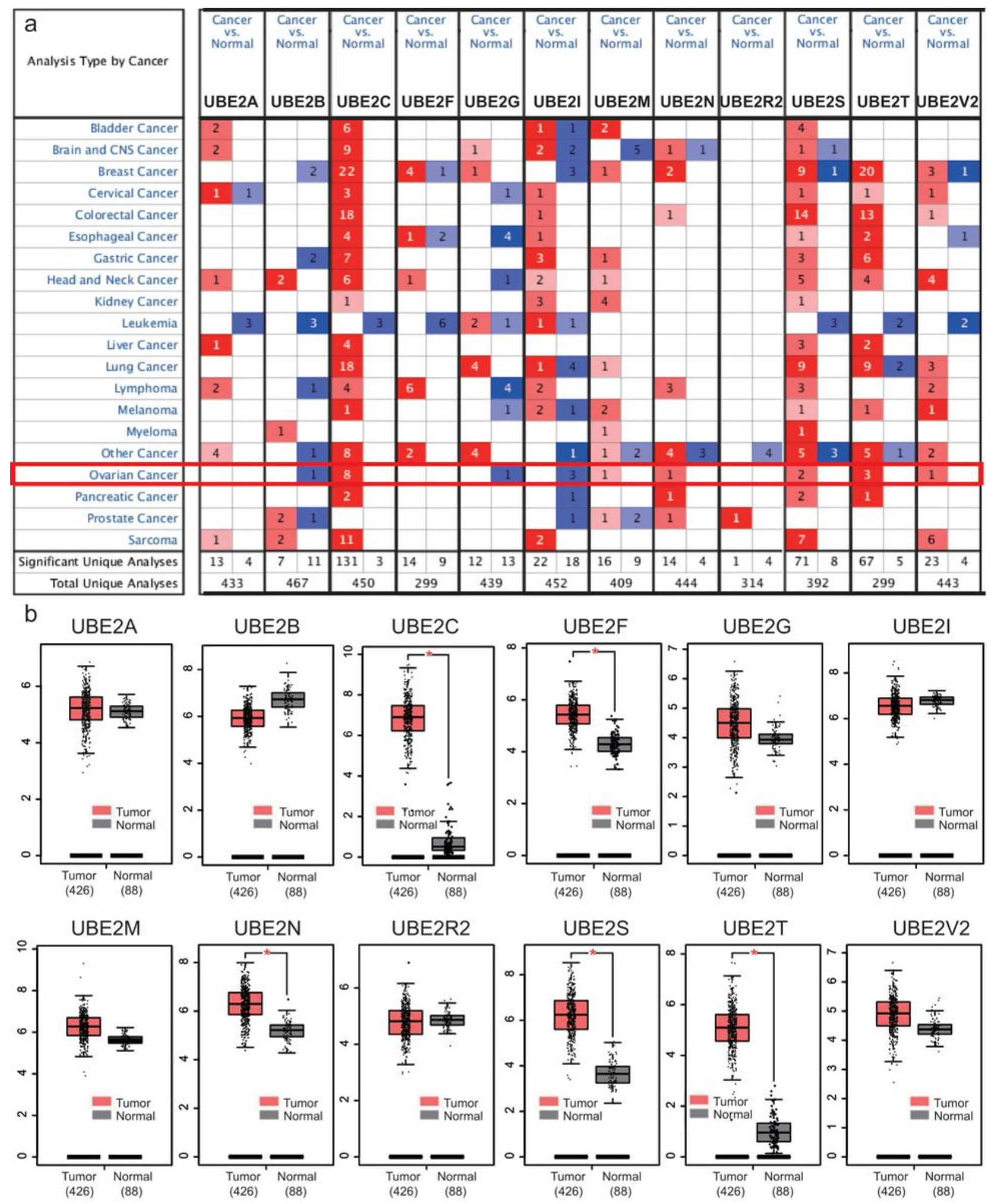

Figure 1 
Differential expression of UBE2s in cancer. a The mRNA expression levels of different UBE2 members in various types of cancers (Oncomine). The numbers in the cells represent the data sets meeting the threshold. Red and blue cells represent upregulation and downregulation, respectively. The color intensity reflects the level of significance. $b$ The mRNA levels of UBE2 members in OC and normal ovarian tissues (GEPIA). The box plots indicate UBE2 expression in 426 cases of OC (red) and 88 cases of corresponding normal tissues (gray). ${ }^{*} \mathrm{P}<0.01$

a

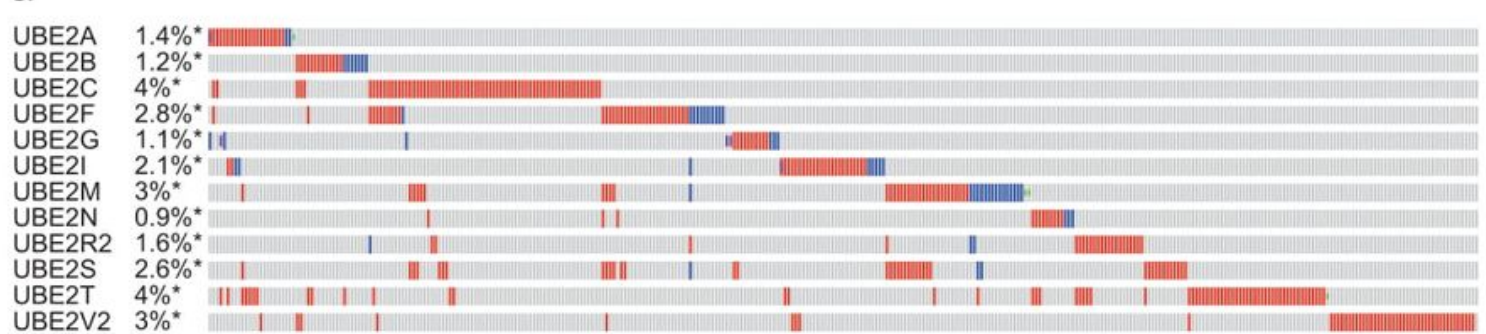

UBE2V2

b
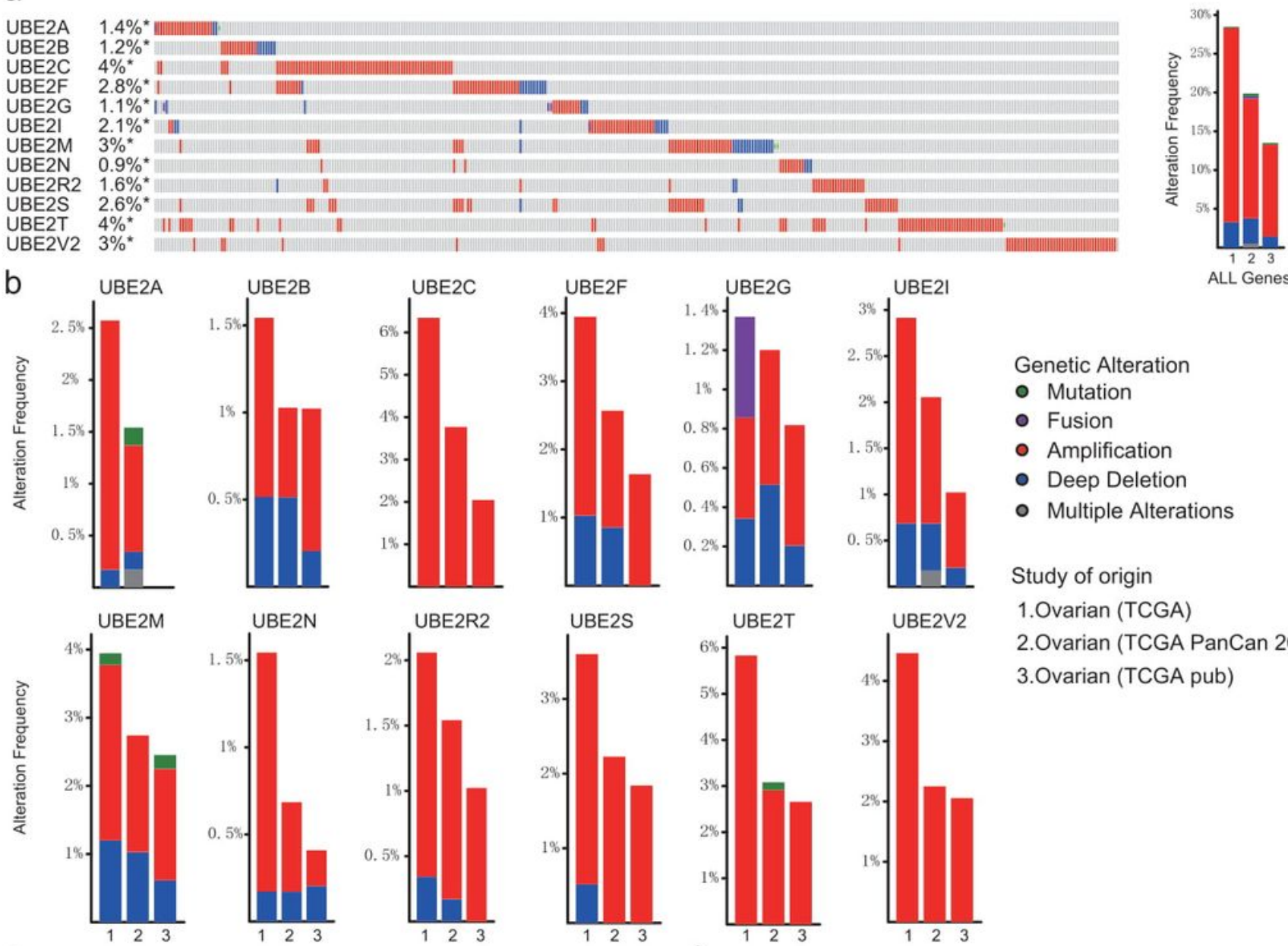

C
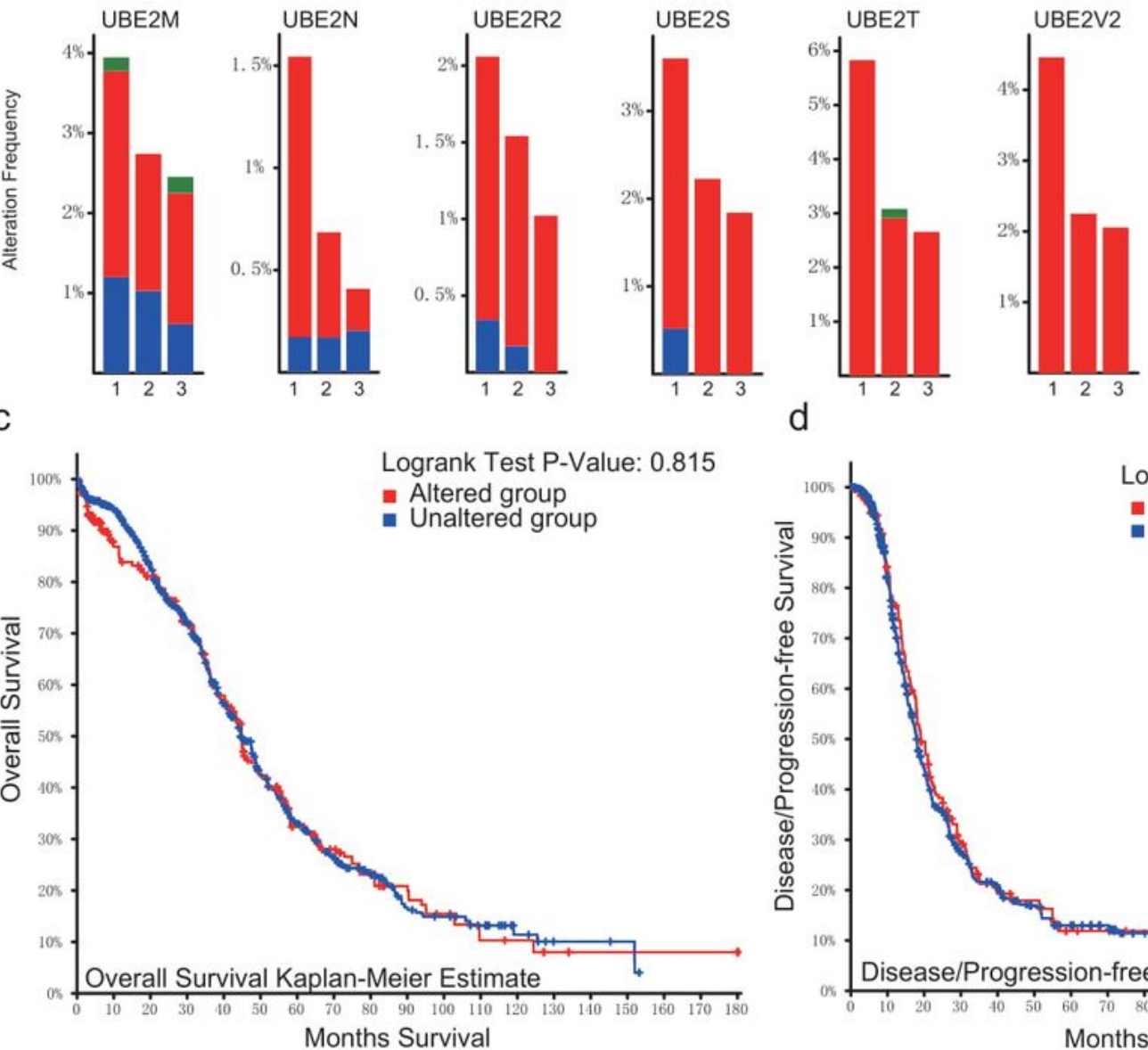

Genetic Alteration

- Mutation

- Fusion

- Amplification

- Deep Deletion

- Multiple Alterations

Study of origin

1.Ovarian (TCGA)

2. Ovarian (TCGA PanCan 2018)

3.Ovarian (TCGA pub)

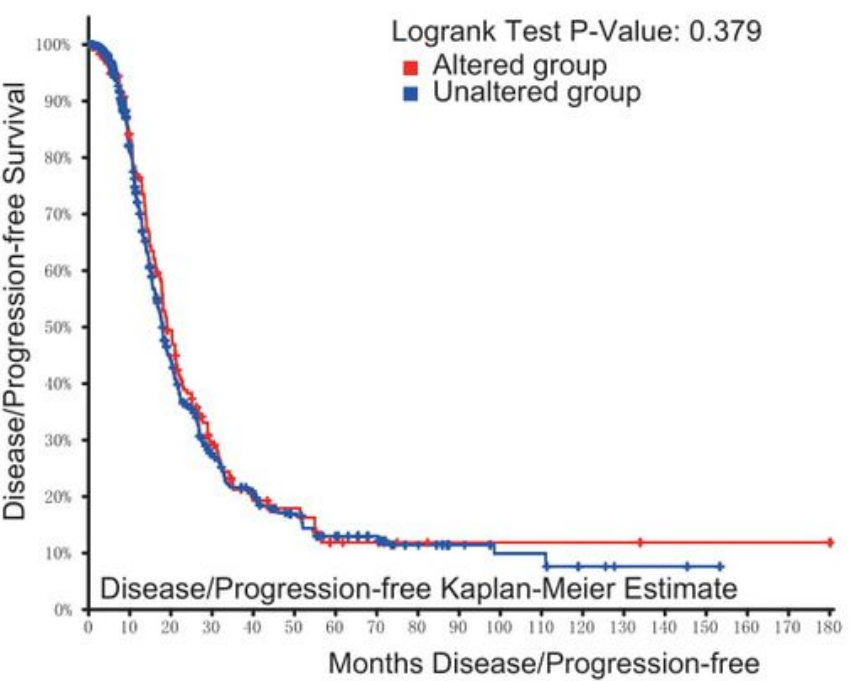

Figure 2 
Frequency of UBE2 gene alterations in OC (cBioPortal). a Left, OncoPrint visual summary of alterations in UBE2 family members; right, summary of genetic alteration frequency in UBE2 genes. b Analysis of genetic alteration frequency in UBE2 family members in three datasets (TCGA, TCGA PanCan, and TCGA pub). The alterations included amplification (red), mutations (green), fusions (purple), deep deletions (blue), and multiple alterations (grey). c Kaplan-Meier plots comparing OS in cases with and without UBE2 gene alterations. d Kaplan-Meier plots comparing DFS in cases with and without UBE2 gene alterations
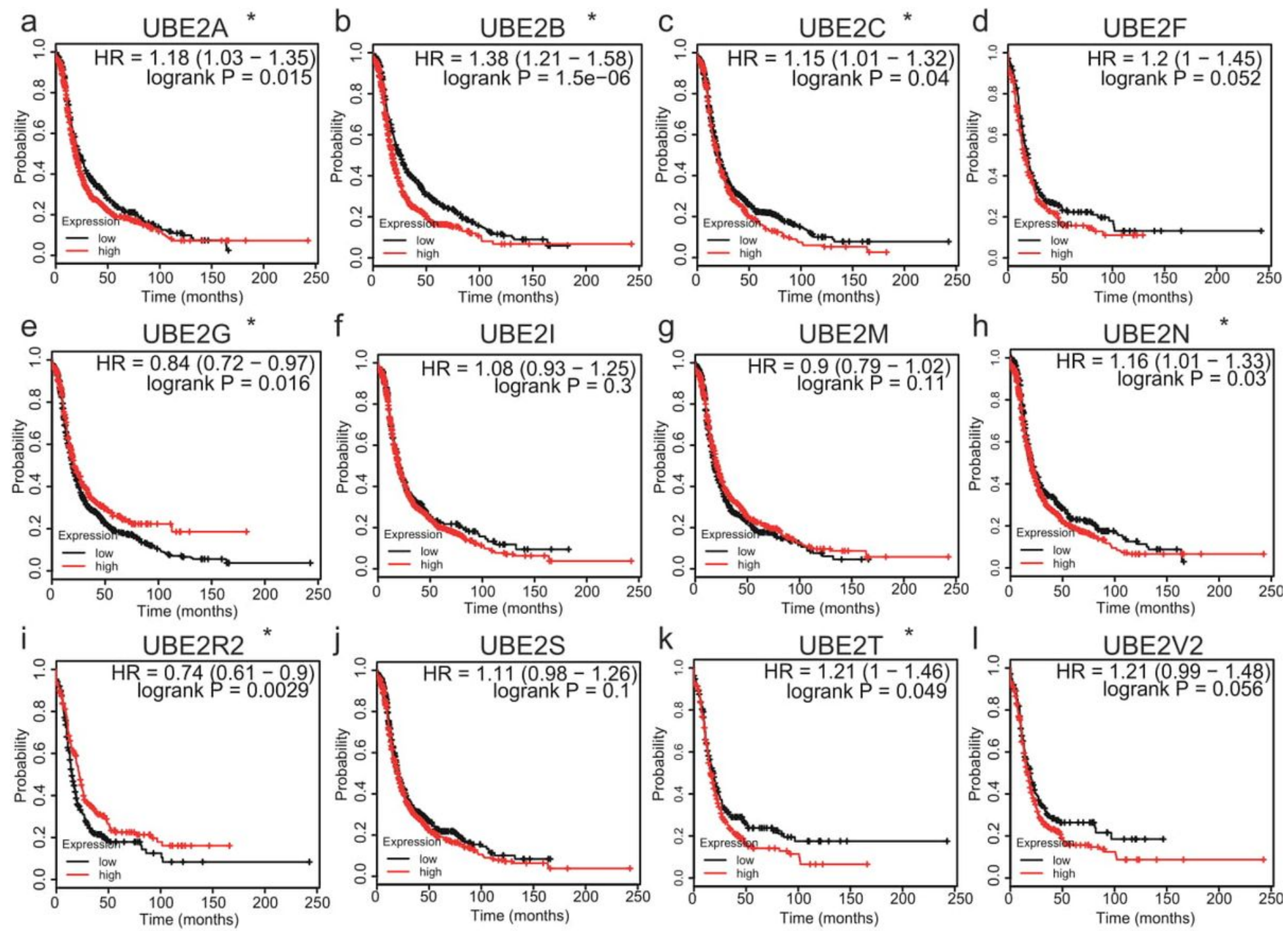

\section{Figure 3}

Prognostic value of UBE2 mRNA levels in patients with OC (PFS in Kaplan-Meier plotter). . a-I Prognostic significance of individual UBE2 members in OC. Red line means high expression and black line means low expression. The $\mathrm{P}$ values were calculated using the log-rank test. ${ }^{*} \mathrm{P}<0.05$ 

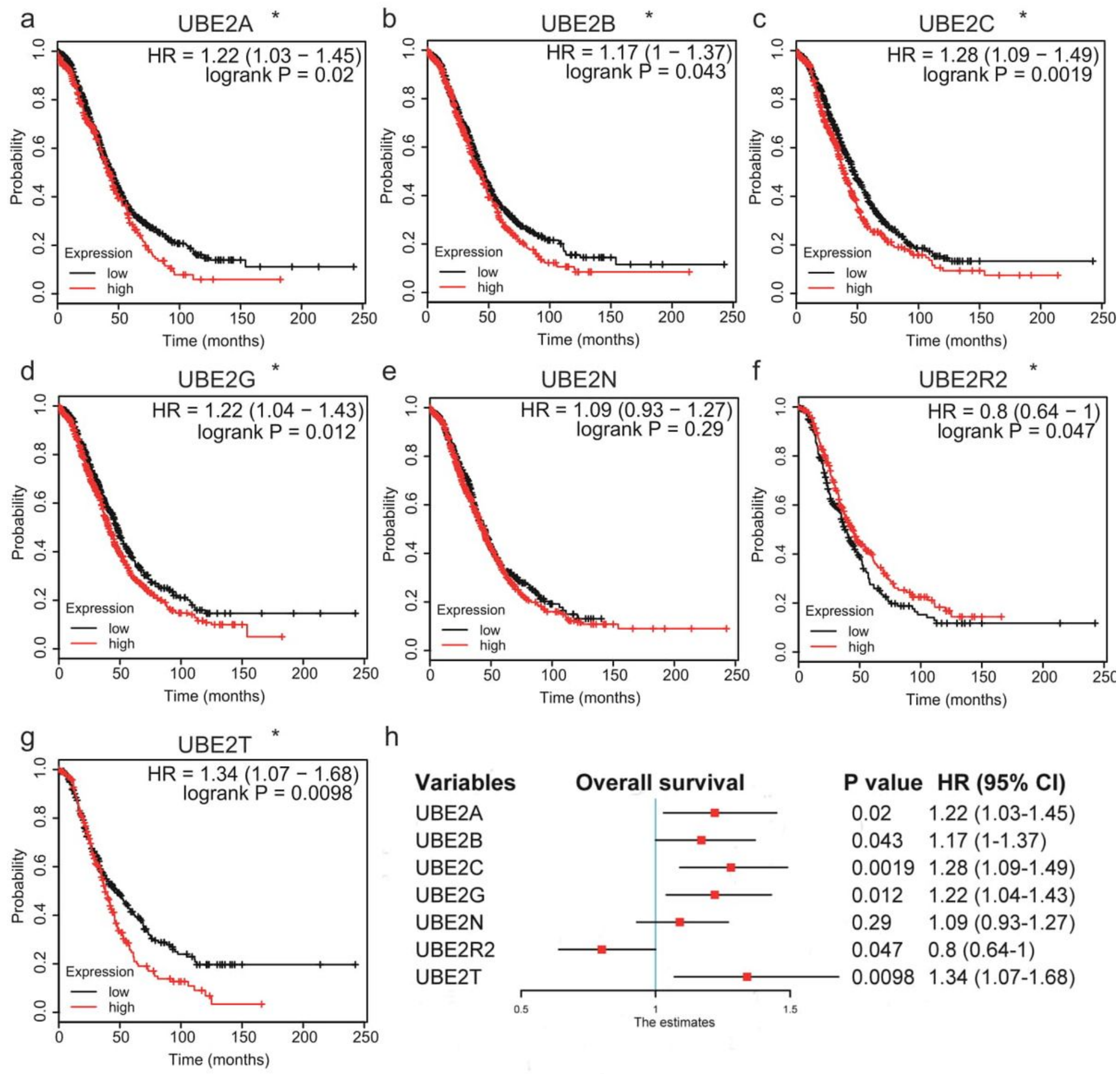

h

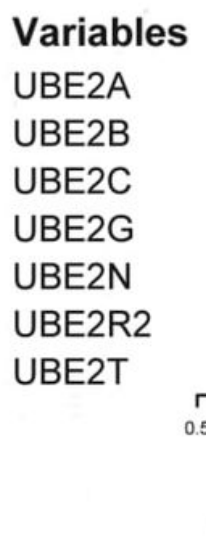

$P$ value $\mathrm{HR}(\mathbf{9 5} \% \mathrm{Cl})$

$0.02 \quad 1.22(1.03-1.45)$

$0.043 \quad 1.17(1-1.37)$

$0.00191 .28(1.09-1.49)$

$0.012 \quad 1.22(1.04-1.43)$

$0.29 \quad 1.09(0.93-1.27)$

$0.047 \quad 0.8(0.64-1)$

$0.00981 .34(1.07-1.68)$

Figure 4

Prognostic value of UBE2 mRNA levels in patients with ovarian serous tumors (OS in Kaplan-Meier plotter). a-g Prognostic significance of individual UBE2s in ovarian serous tumors. h Prognostic HRs of individual UBE2s in ovarian serous tumors. Red line means high expression and black line means low expression. The $\mathrm{P}$ values were calculated using the log-rank test. ${ }^{*} \mathrm{P}<0.05$ 

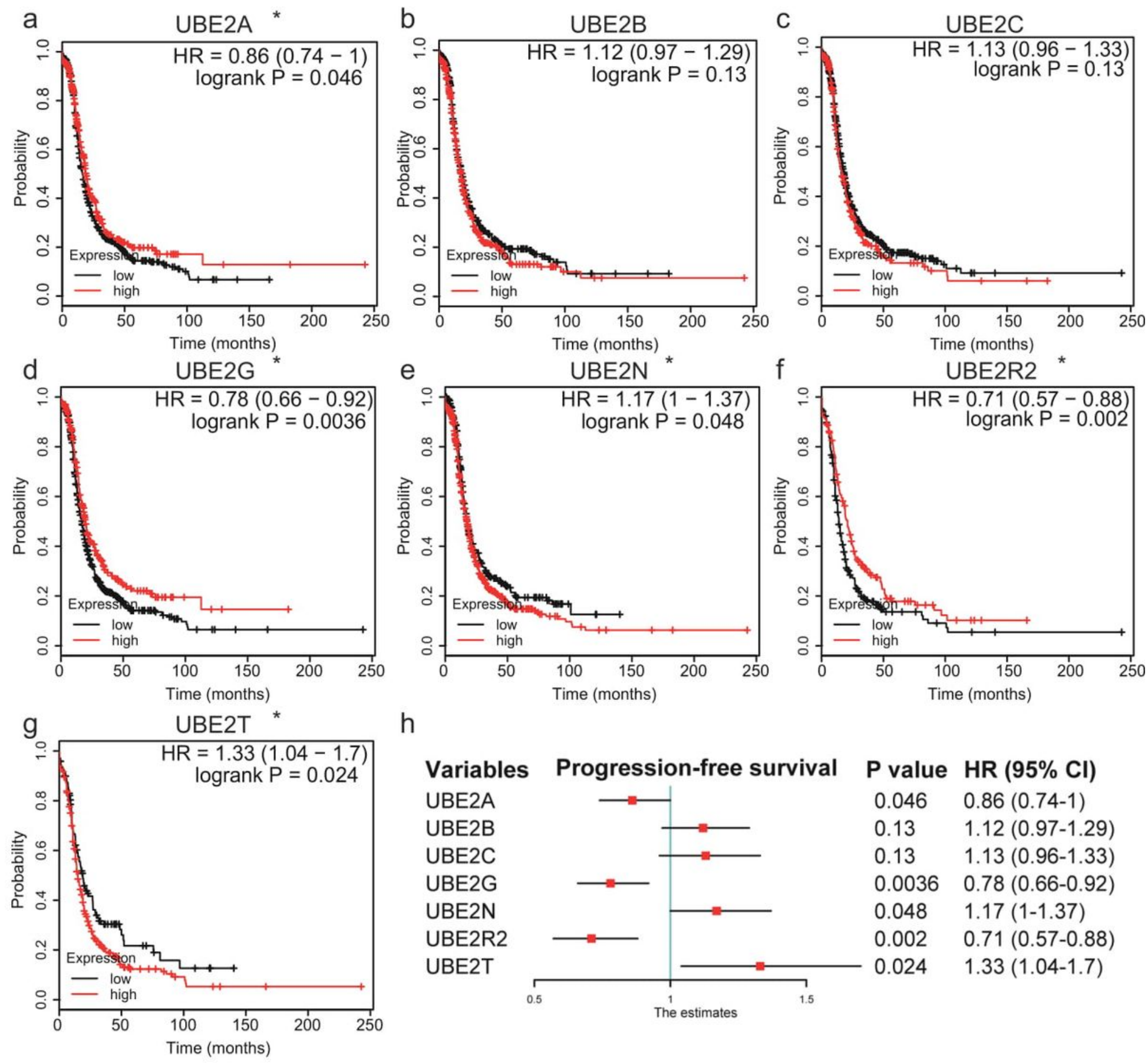

h

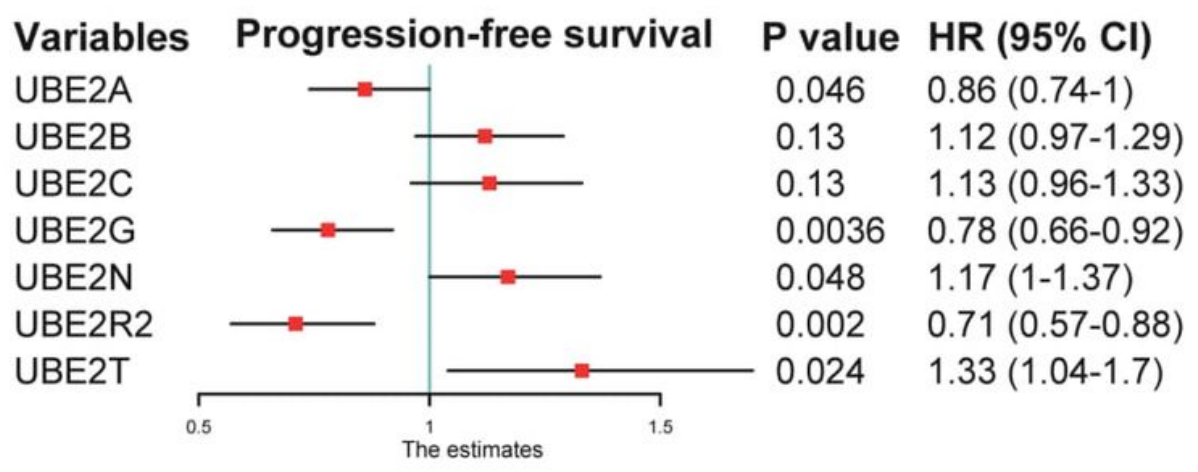

Figure 5

Prognostic value of UBE2 mRNA levels in patients with ovarian serous tumors (PFS in Kaplan-Meier plotter). a-g Prognostic significance of individual UBE2s in ovarian serous tumors. h Prognostic HRs of individual UBE2s in ovarian serous tumors. Red line means high expression and black line means low expression. The $\mathrm{P}$ values were calculated using the log-rank test. ${ }^{*} \mathrm{P}<0.05$ 
a

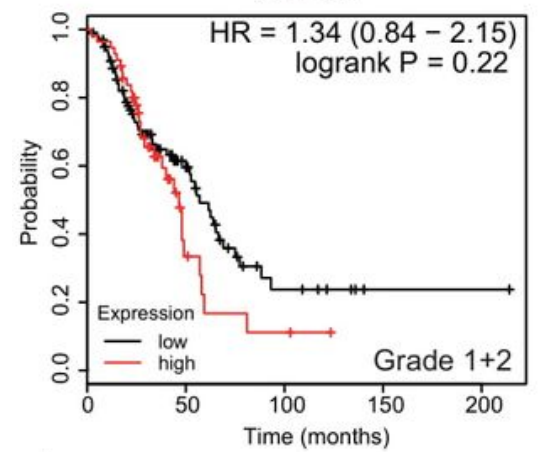

d

UBE2T

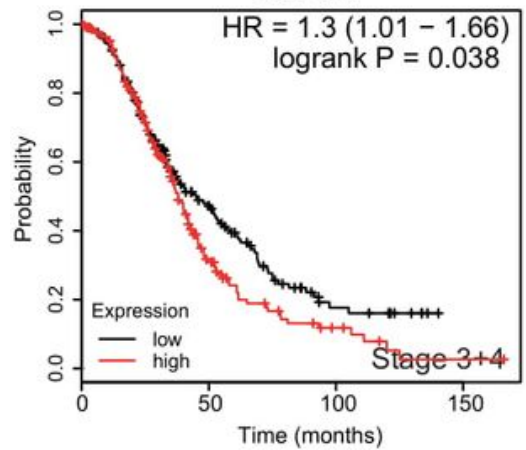

b

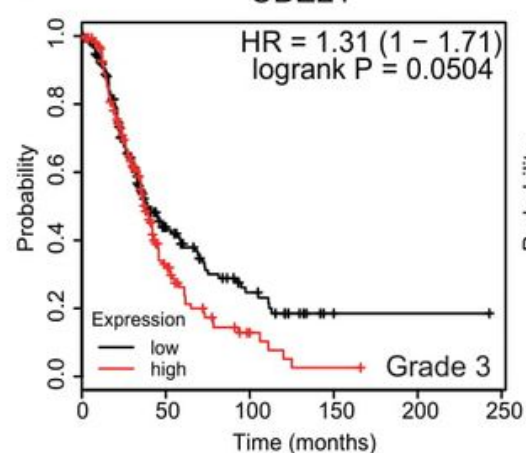

e

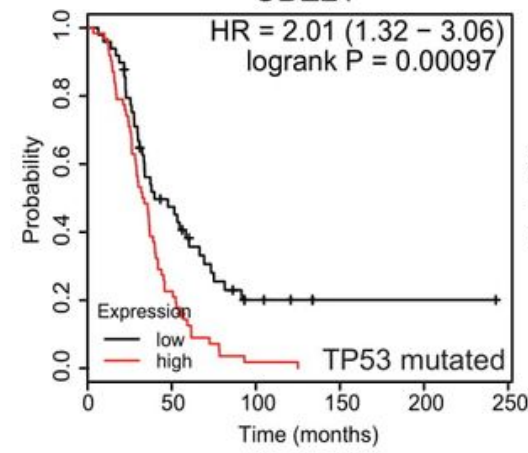

C

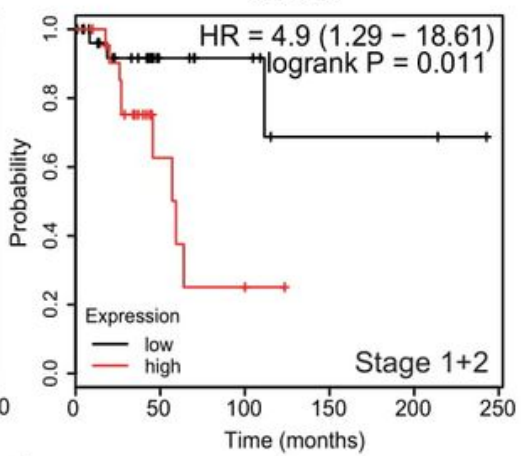

$f$

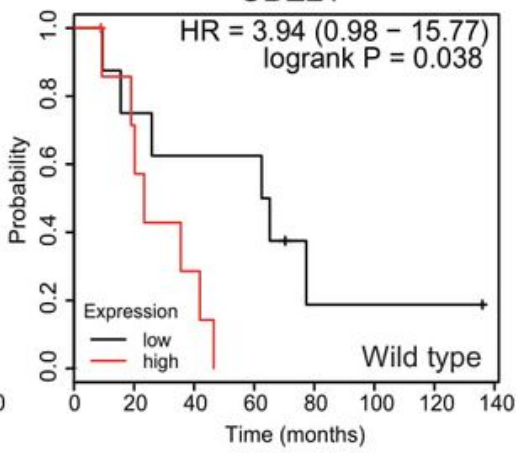

g

\begin{tabular}{|c|c|c|c|c|c|c|c|c|c|c|c|c|c|}
\hline & UBE2A & UBE2B & UBE2C & UBE2F & UBE2G & UBE2I & UBE2M & UBE2N & UBE2R2 & UBE2S & UBE2T & UBE2V2 & \\
\hline UBE2A & 1 & 0.27 & 0.25 & 0.34 & 0.46 & 0.28 & 0.26 & 0.47 & 0.38 & 0.4 & 0.43 & 0.42 & \\
\hline UBE2B & 0.27 & & -0.017 & 0.18 & 0.17 & 0.11 & 0.013 & 0.14 & 0.066 & 0.02 & 0.12 & 0.18 & \\
\hline UBE2C & 0.25 & -0.017 & 1 & 0.2 & 0.14 & 0.17 & 0.26 & 0.35 & 0.28 & 0.67 & 0.66 & 0.35 & \\
\hline UBE2F & 0.34 & 0.18 & 0.2 & 1 & 0.31 & 0.22 & 0.2 & 0.36 & 0.22 & 0.19 & 0.24 & 0.33 & \\
\hline UBE2G & 0.46 & 0.17 & 0.14 & 0.31 & 1 & 0.37 & 0.3 & 0.49 & 0.5 & 0.19 & 0.31 & 0.58 & \\
\hline UBE2I & 0.28 & 0.11 & 0.17 & 0.22 & 0.37 & 1 & 0.35 & 0.36 & 0.37 & 0.087 & 0.28 & 0.35 & 1 \\
\hline UBE2M & 0.26 & 0.013 & 0.26 & 0.2 & 0.3 & 0.35 & 1 & 0.24 & 0.3 & 0.58 & 0.24 & 0.32 & \\
\hline UBE2N & 0.47 & 0.14 & 0.35 & 0.36 & 0.49 & 0.36 & 0.24 & 1 & 0.39 & 0.32 & 0.54 & 0.51 & \\
\hline UBE2R2 & 0.38 & 0.066 & 0.28 & 0.22 & 0.5 & 0.37 & 0.3 & 0.39 & 1 & 0.26 & 0.33 & 0.43 & 0.5 \\
\hline UBE2S & 0.4 & 0.02 & 0.67 & 0.19 & 0.19 & 0.087 & 0.58 & 0.32 & 0.26 & 1 & 0.56 & 0.32 & \\
\hline UBE2T & 0.43 & 0.12 & 0.66 & 0.24 & 0.31 & 0.28 & 0.24 & 0.54 & 0.33 & 0.56 & 1 & 0.54 & \\
\hline UBE2V2 & 0.42 & 0.18 & 0.35 & 0.33 & 0.58 & 0.35 & 0.32 & 0.51 & 0.43 & 0.32 & 0.54 & & 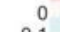 \\
\hline
\end{tabular}

h

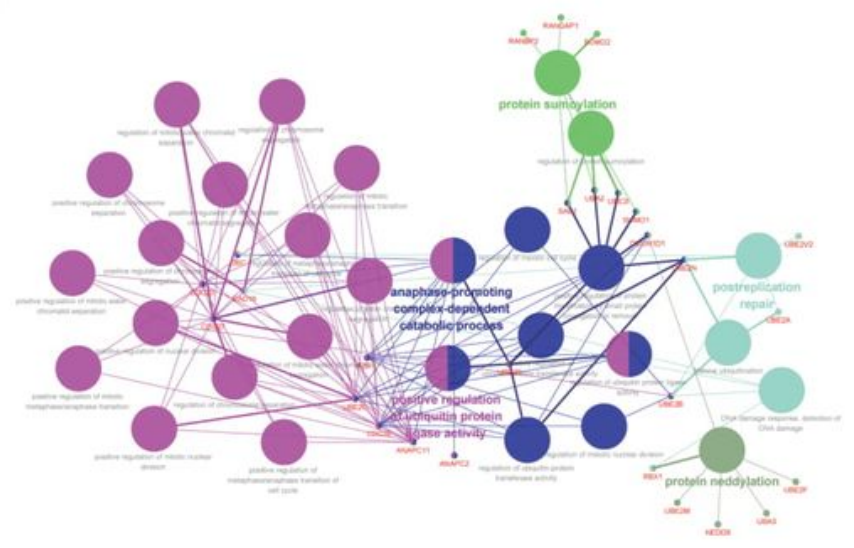

i

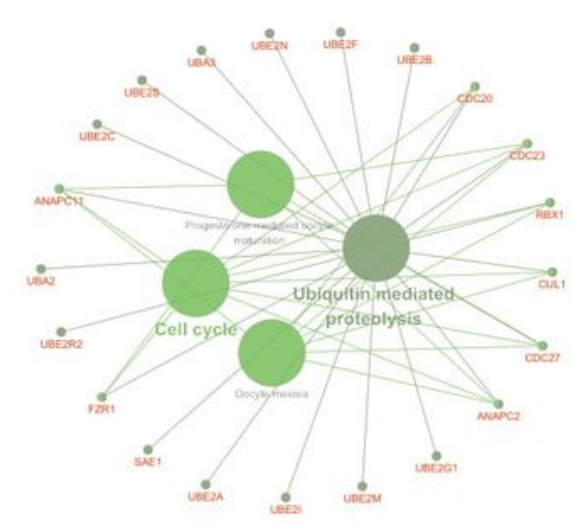

\section{Figure 6}

Prognostic significance of UBE2T expression level in ovarian serous tumors of different pathological grade $(a, b)$, FIGO stage (c,d), and TP53 mutational status (e,f) (OS in Kaplan-Meier plotter). Analysis of the interactions between UBE2 family members (GEPIA and Cytoscape). g Spearman' s correlation coefficients between UBE2 family members. Red and blue cells represent positive and negative correlations, respectively. The color intensity reflects the strength of the correlations. $\mathrm{h}$ GO biological 
process enrichment analysis of UBE2s and their interactors. i KEGG pathway analysis of UBE2s and their interactors. ${ }^{*} \mathrm{P}<0.05$

a

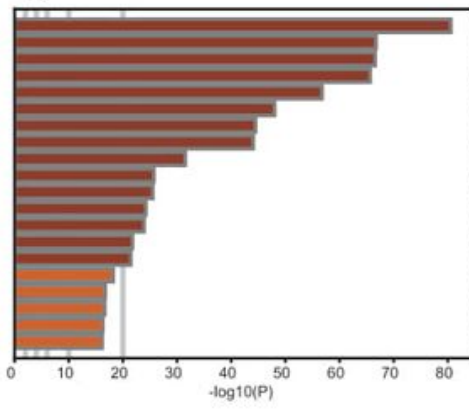

C

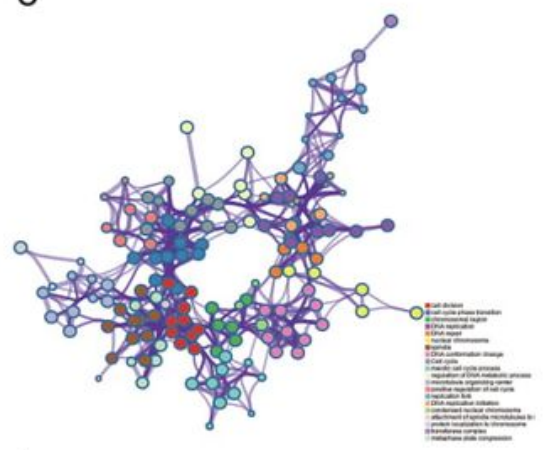

f

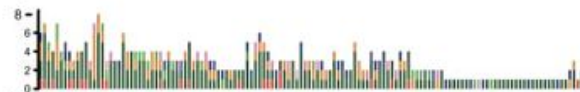

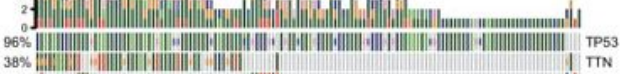

${ }_{9 \%}^{11 \%}$ || || |||

\begin{tabular}{ll|}
$9 \%$ & 11 \\
$8 \%$ & III
\end{tabular}

${ }^{8 \%}$ || | | | || || ||

(1 I II

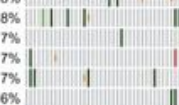

$6 \%$ | || || ||

$\mathrm{h}$

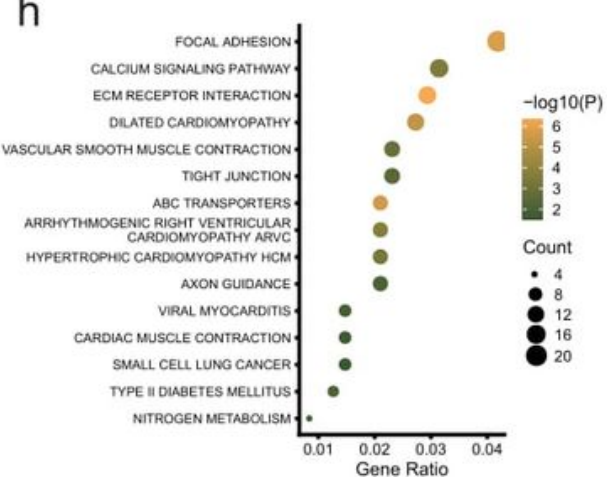

b

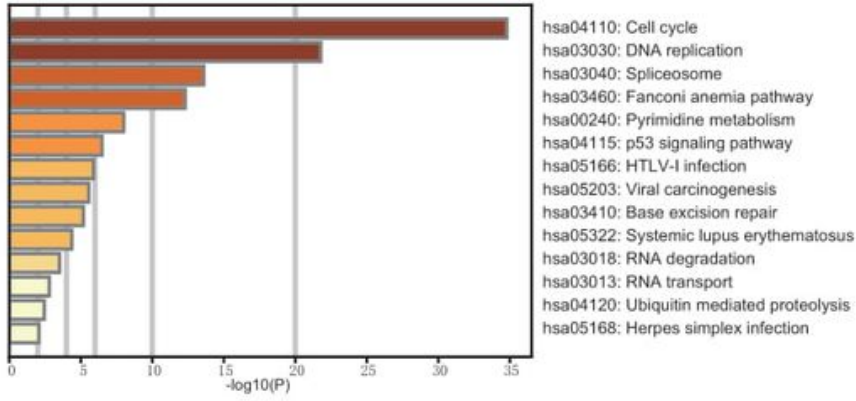

e

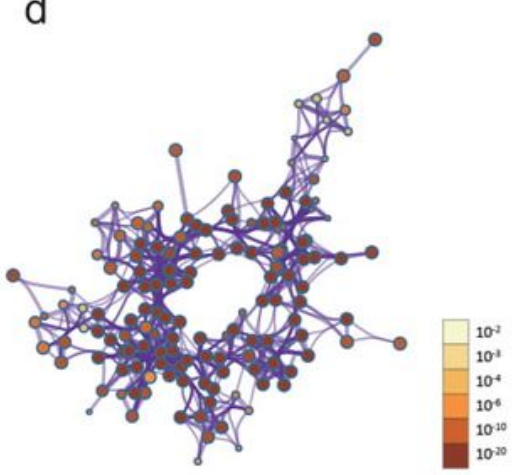

g
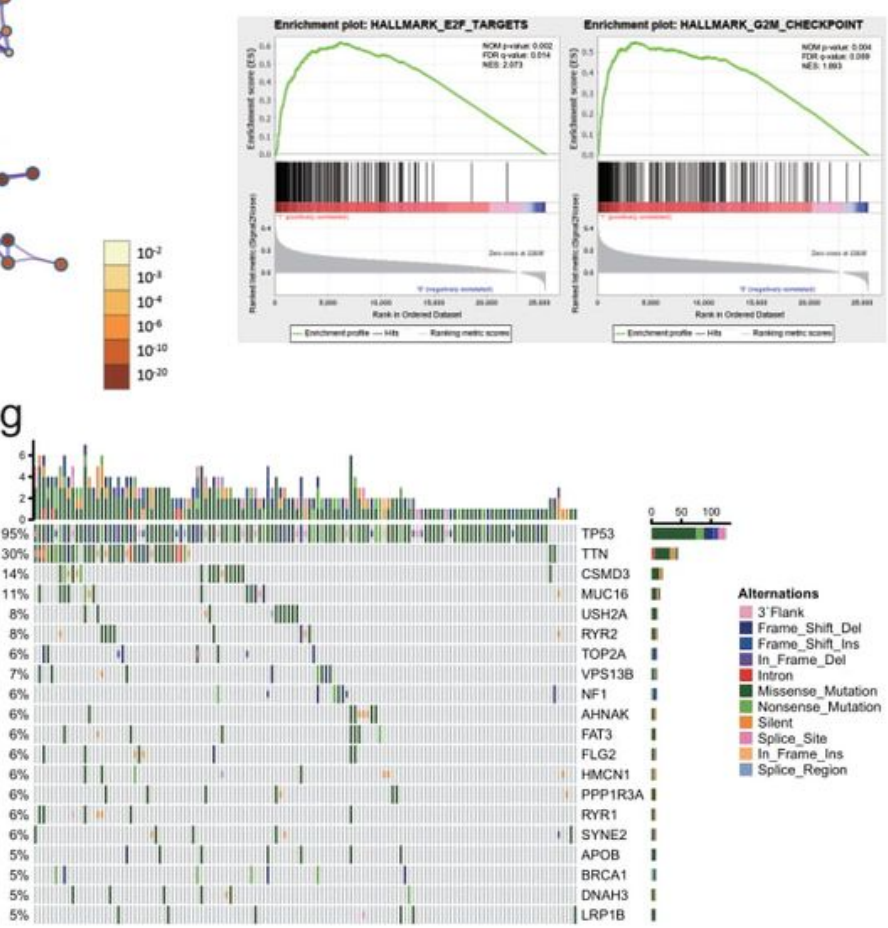

i
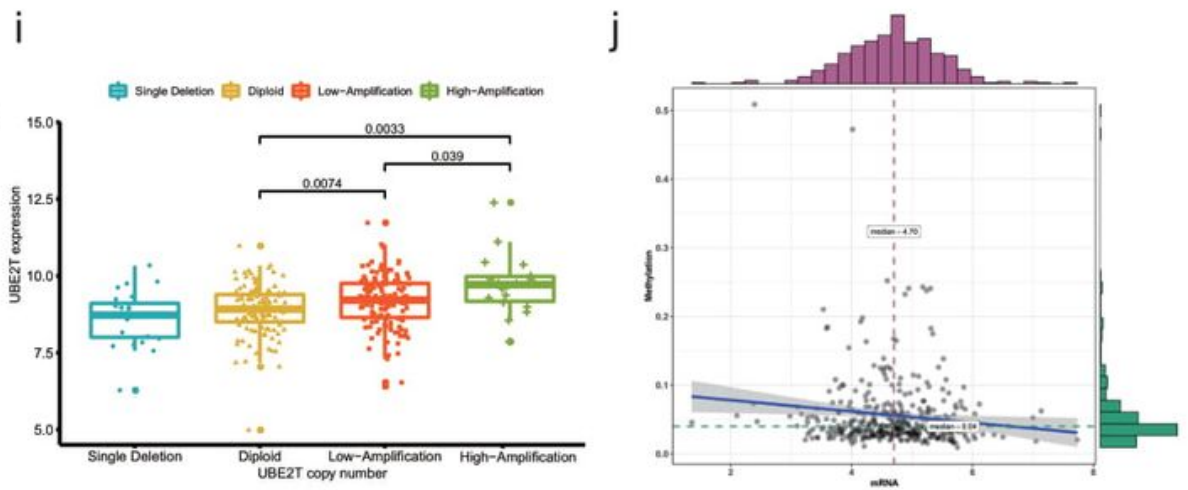

Figure 7

Molecular mechanisms related to UBE2T expression in ovarian cancer (Metascape). a Bar graph of GO enriched terms colored by P-values. b Bar graph of KEGG enriched terms colored by P-values. c Network of enriched terms colored according to clusters: terms belonging to the same cluster are more closely 
related to each other. $d$ Network of enriched terms colored according to P-value: the higher the number of genes in each term, the higher the statistical significance. e Gene sets significantly enriched in patients with high UBE2T expression (HALLMARK_E2F_TARGETS and HALLMARK_G2M_CHECKPOINT). $f$ Top 20 somatically mutated genes in patients with high UBE2T expression. $g$ Top 20 somatically mutated genes in patients with low UBE2T expression. $\mathrm{h}$ KEGG pathway analysis in the UBE2T high expression mutation group. i Analysis of the correlation between copy number variation and UBE2T expression. j Analysis of the correlation between methylation and UBE2T expression

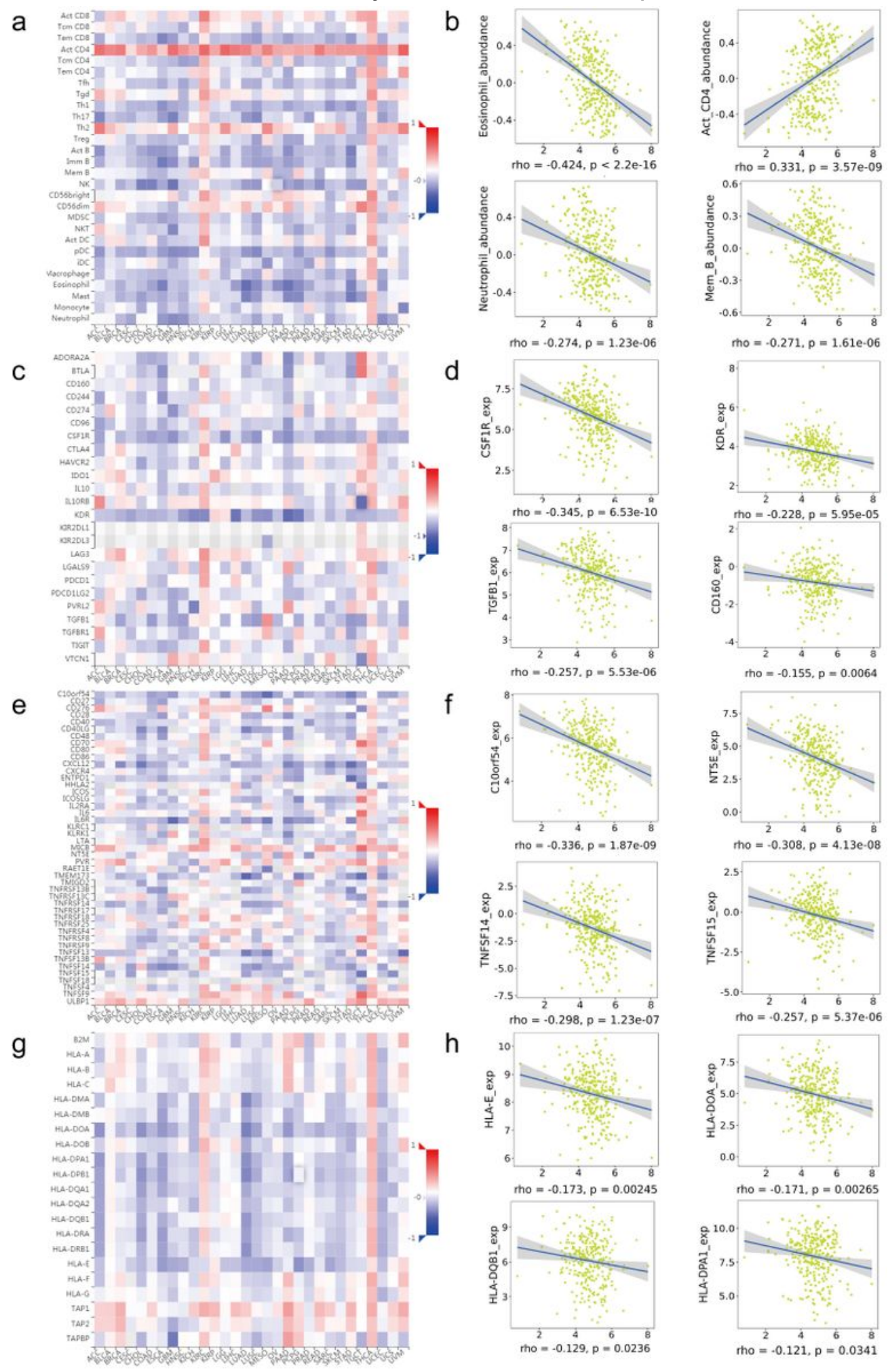




\section{Figure 8}

The correlation between immune factors and UBE2T in ovarian cancer determined by TISIDB analysis. a Correlation between UBE2T expression and tumor-infiltrating lymphocytes (TILs). b TILs with the highest correlation with UBE2T expression. c Correlation between UBE2T expression and immune inhibitors. $d$ Immune inhibitors showing the highest correlation with UBE2T expression. e Correlation between UBE2T expression and immune stimulators. $f$ Immune stimulators showing the highest correlation with UBE2T expression. g Correlation between UBE2T expression and MHC molecules. h MHC molecules exhibiting the highest correlation with UBE2T expression. Red and blue cells indicate positive and negative correlations, respectively. The color intensity reflects the strength of the correlation. 
a

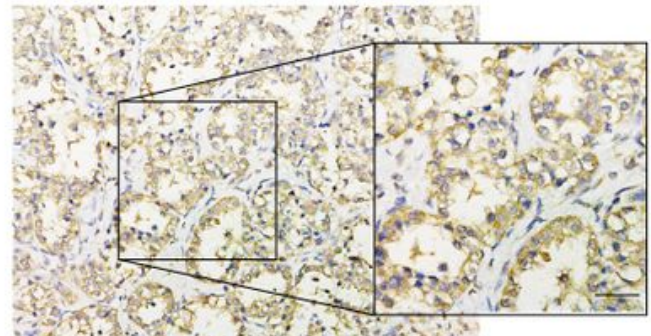

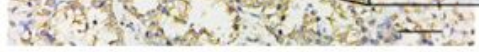

C

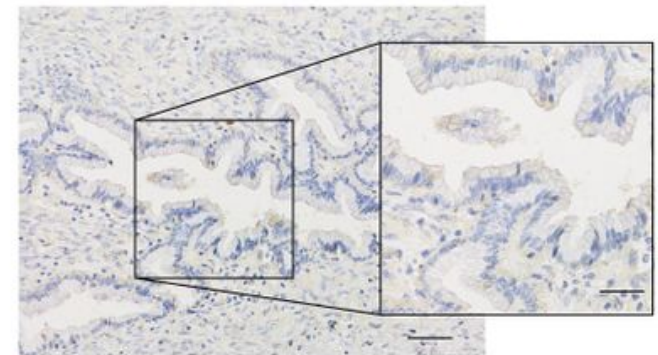

b

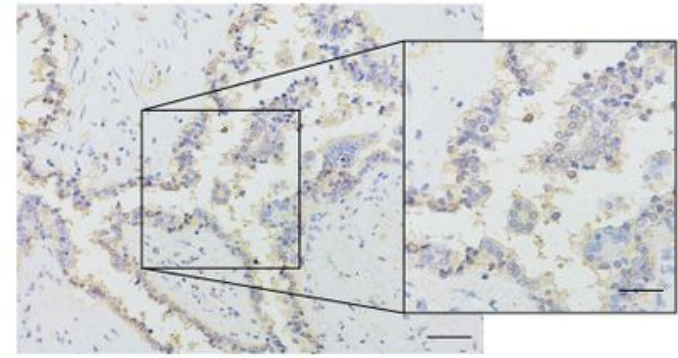

d
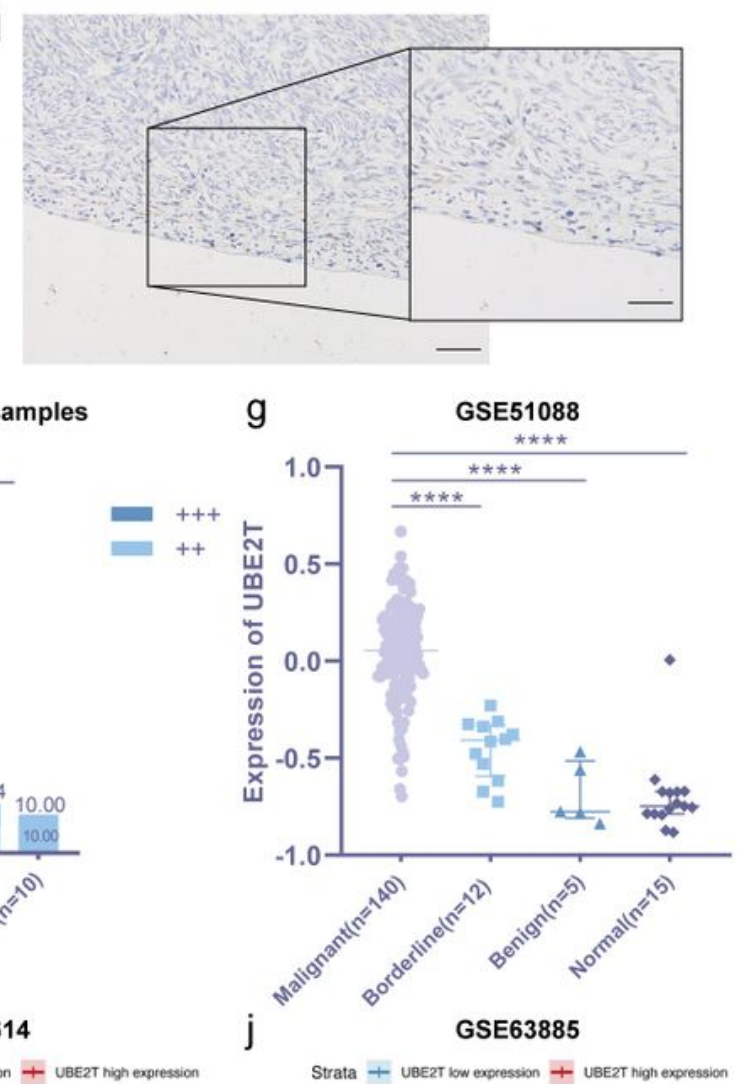

Strata + UBE2T low expression + UBE2T high expression

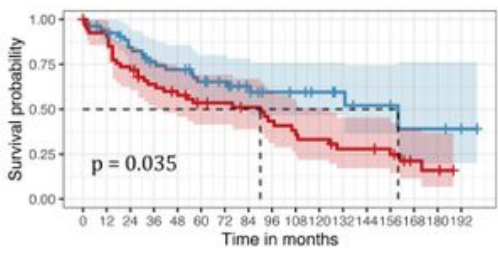

Percentage at risk (\%):

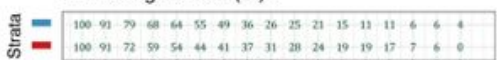

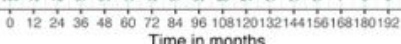

Number of censoring

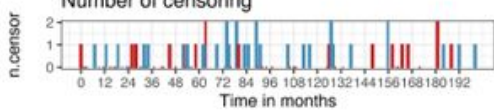

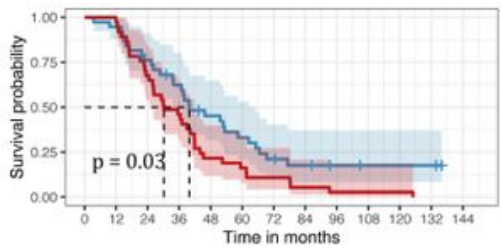

Percentage at risk (\%):

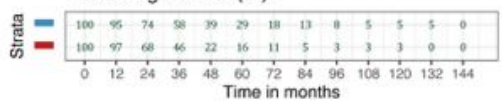

Number of censoring

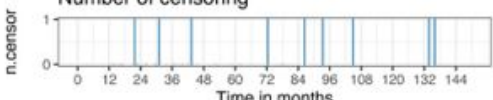

\section{Figure 9}

UBE2T expression in different ovarian tissues. Immunohistochemical detection of UBE2T in OC tissues (a), borderline tumors (b), benign tumors (c), and normal ovarian tissues (d) (magnification, left $\times 200$, right $\times 400$ ). e Positive rates of UBE2T expression in clinical samples of different groups. $f$ Highly positive rates of UBE2T expression in clinical samples of different groups. g UBE2T expression in the different groups, according to the GSE51088 dataset. h Correlation between UBE2T expression and overall survival 
in 86 patients, based on clinical samples. i Correlation between UBE2T expression and overall survival in 107 samples, according to the GSE73614 dataset. j Correlation between UBE2T expression and overall survival in 75 samples, according to the GSE63885 dataset. ${ }^{*} P<0.05$, ${ }^{*} P<0.01,{ }^{\star \star *} P<0.001$, ${ }^{\star \star \star *} P<0.0001$

\section{Supplementary Files}

This is a list of supplementary files associated with this preprint. Click to download.

- Additionalfile3FigureS1.tif

- Additionalfile2.xlsx

- Additionalfile1.xls 